\title{
Cobalt-Catalyzed Reductive Coupling of Saturated Alkyl Halides with Activated Alkenes
}

\author{
Paritosh Shukla, Yun Chu Hsu and Chien-Hong Cheng* \\ chcheng@mx.nthu.edu.tw
}

Department of Chemistry, National Tsing Hua University, Hsinchu, 30013, Taiwan

\section{SUPPORTING INFORMATION}

\section{List of Contents}

1. General Synthetic Methods $\quad$ S2

2. Spectral Data for All the Compounds S2-S5

3. References S6

4. $\quad{ }^{1} \mathrm{HNMR},{ }^{13} \mathrm{C}$ and DEPT Spectra of Selected Compounds $\quad$ S7-S32 
Procedure for the Reductive Coupling of (2-Bromoethyl)benzene 1a with Methyl Acrylate 2a. A sealed tube $(20 \mathrm{~mL})$ containing $\mathrm{CoI}_{2}(\mathrm{dppe})(0.10 \mathrm{mmol})$ and zinc powder ( $2.5 \mathrm{mmol}$ ) was evacuated and purged with nitrogen three times. Freshly distilled $\mathrm{CH}_{3} \mathrm{CN}$ (2.5 mL), (2-bromoethyl)benzene $\mathbf{1 a}(1.0 \mathrm{mmol})$, methyl acrylate $\mathbf{2 a}(4.0 \mathrm{mmol})$ and $\mathrm{H}_{2} \mathrm{O}$ $(1.0 \mathrm{mmol})$ were added to the sealed tube via syringes. The reaction mixture was heated with stirring at $80{ }^{\circ} \mathrm{C}$ for $12 \mathrm{~h}$ and was then cooled, diluted with dichloromethane and stirred in the air for $10 \mathrm{~min}$. The mixture was filtered through a Celite and silica gel pad and washed with dichloromethane. The filtrate was concentrated and the residue was purified on a silica gel column using hexane-ethyl acetate as eluent to afford the desired product 3a.

Products 3b-m were prepared according to similar procedure except when the iodo compounds were used the reaction time was reduced to $6 \mathrm{~h}$ and only $5 \mathrm{~mol} \%$ of $\mathrm{CoI}_{2}$ (dppe) and 2-3 equiv of acrylate (2.0-3.0 mmol) was employed. Compounds $\mathbf{3 a},{ }^{1}$ $\mathbf{3 b},{ }^{2} \mathbf{3 c},{ }^{8} \mathbf{3 d},{ }^{3} \mathbf{3 e},{ }^{9} \mathbf{3 f},{ }^{2} \mathbf{3 g},{ }^{6} \mathbf{3 h},{ }^{2} \mathbf{3 j},{ }^{4} \mathbf{3} \mathbf{k}^{5}$ and $3 \mathbf{l}^{7}$ are previously reported. Important spectral data of these compounds are listed below.

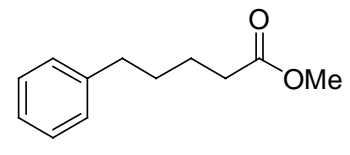

(3a) CAS registry No: $20620-59-1 .{ }^{1} \mathrm{H}$ NMR $\left(400 \mathrm{MHz}, \mathrm{CDCl}_{3}\right): \delta$ $7.26(\mathrm{~m}, 2 \mathrm{H}), 7.16(\mathrm{t}, 3 \mathrm{H}, J=6.4 \mathrm{~Hz}), 3.64(\mathrm{~s}, 3 \mathrm{H}), 2.61(\mathrm{t}, 2 \mathrm{H}, J=6.8 \mathrm{~Hz}), 2.32(\mathrm{t}, 2 \mathrm{H}, J$ $=6.4 \mathrm{~Hz}), 1.63-1.66(\mathrm{~m}, 4 \mathrm{H}) \cdot{ }^{13} \mathrm{C} \mathrm{NMR}\left(400 \mathrm{MHz}, \mathrm{CDCl}_{3}\right): \delta 174.0,142.1,128.3,128.3$, 125.8, 51.4, 35.5, 33.9, 24.5. HRMS calcd for $\mathrm{C}_{12} \mathrm{H}_{16} \mathrm{O}_{2}$ 192.1150; found 192.1155.

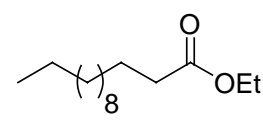

(3b) CAS registry No: 28267-29-0. ${ }^{1} \mathrm{H}$ NMR $\left(400 \mathrm{MHz}, \mathrm{CDCl}_{3}\right): \delta 4.10$ (q, 2H, $J=7.2 \mathrm{~Hz}), 2.27$ (t, 2H, $J=7.0 \mathrm{~Hz}), 1.42-1.66(\mathrm{~m}, 3 \mathrm{H}), 1.19-1.40(\mathrm{~m}, 20 \mathrm{H}), 0.87$ (t, 3H, $J=6.8 \mathrm{~Hz}) .{ }^{13} \mathrm{C}$ NMR $\left(400 \mathrm{MHz}, \mathrm{CDCl}_{3}\right): \delta 174.1,60.3,34.6,32.1,29.8,29.7$, 29.6 (3C), 29.5, 29.4, 25.2, 22.9, 14.5, 14.3. HRMS calcd for $\mathrm{C}_{15} \mathrm{H}_{30} \mathrm{O}_{2}$ 242.2246; found 242.2243 . 


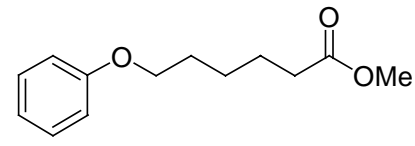

(3c) CAS registry No: $92156-72-4 .{ }^{1} \mathrm{H}$ NMR $\left(400 \mathrm{MHz}, \mathrm{CDCl}_{3}\right)$ : $\delta$ 7.24-7.30 (m, 2H), 6.88-6.95 (m, 3H), $3.95(\mathrm{t}, 2 \mathrm{H}, J=6.6 \mathrm{~Hz}), 3.68(\mathrm{~s}, 3 \mathrm{H}), 2.31(\mathrm{t}, 2 \mathrm{H}$, $J=7.2 \mathrm{~Hz}), 1.75(\mathrm{~m}, 2 \mathrm{H}),, 1.68(\mathrm{~m}, 2 \mathrm{H}),, 1.51(\mathrm{~m}, 2 \mathrm{H},) .{ }^{13} \mathrm{C} \mathrm{NMR}\left(400 \mathrm{MHz}, \mathrm{CDCl}_{3}\right): \delta$ 173.9, 158.9, 129.3, 120.4, 114.4, 67.4, 51.4, 33.9, 28.9, 25.6, 24.6. HRMS calcd for $\mathrm{C}_{13} \mathrm{H}_{18} \mathrm{O}_{3} 222.1256$; found 222.1251.

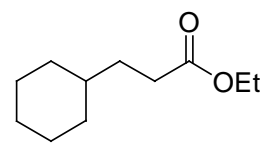

(3d) CAS registry No: 3289-28-9. ${ }^{1} \mathrm{H}$ NMR $\left(400 \mathrm{MHz}, \mathrm{CDCl}_{3}\right): \delta 4.08$ (q, $2 \mathrm{H}, J=7.2 \mathrm{~Hz}$ ), 2.26 (t, $2 \mathrm{H}, J=7.0 \mathrm{~Hz}), 1.59-1.67(\mathrm{~m}, 5 \mathrm{H}), 1.47$ (m, 2H), 1.08-1.28 (m, 7H), 0.81-0.89 (m, 2H). ${ }^{13} \mathrm{C}$ NMR (400 MHz, $\left.\mathrm{CDCl}_{3}\right): \delta 174.4,60.4,37.4,33.2(2 \mathrm{C})$, 32.6, 32.2, 26.7, 26.4(2C), 14.5. HRMS calcd for $\mathrm{C}_{11} \mathrm{H}_{20} \mathrm{O}_{2} 184.1463$; found 184.1465

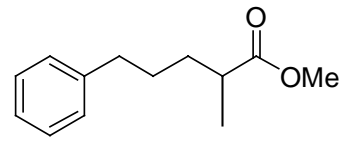

(3e) CAS registry No: 59339-36-5. ${ }^{1} \mathrm{H}$ NMR $\left(400 \mathrm{MHz}, \mathrm{CDCl}_{3}\right): \delta$ 7.22-7.28 (m, 2H), 7.13-7.18 (m, 3H), 3.63 (s, 3H), 2.62 (t, 2H, $J=6.4 \mathrm{~Hz}), 2.43(\mathrm{~m}$, $1 \mathrm{H},), 1.57-1.69(\mathrm{~m}, 3 \mathrm{H}), 1.42-1.49(\mathrm{~m}, 1 \mathrm{H}), 1.13(\mathrm{~d}, 3 \mathrm{H}, J=6.2 \mathrm{~Hz}) .{ }^{13} \mathrm{C}$ NMR $(400$ $\left.\mathrm{MHz}, \mathrm{CDCl}_{3}\right): \delta 177.1,142.1,125.7,128.3,51.4,39.3,35.7,33.5,29.0,17.0$. HRMS calcd for $\mathrm{C}_{13} \mathrm{H}_{18} \mathrm{O}_{2}$ 206.1307; found 206.1311 .

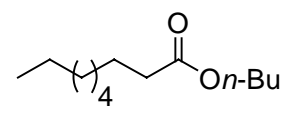

(3f) CAS registry No: 50623-57-9. ${ }^{1} \mathrm{H}$ NMR $\left(400 \mathrm{MHz}, \mathrm{CDCl}_{3}\right): \delta 4.0(\mathrm{t}$, $2 \mathrm{H}, J=6.8 \mathrm{~Hz}), 2.26(\mathrm{t}, 2 \mathrm{H}, J=7.2 \mathrm{~Hz}), 1.56-1.61(\mathrm{~m}, 4 \mathrm{H}), 1.32-1.38(\mathrm{~m}, 2 \mathrm{H}), 1.24-1.31$ (m, 14H), $0.91(\mathrm{t}, 3 \mathrm{H}, J=7.0 \mathrm{~Hz}), 0.85(\mathrm{t}, 3 \mathrm{H}, J=7.2 \mathrm{~Hz}) .{ }^{13} \mathrm{C} \mathrm{NMR}\left(400 \mathrm{MHz}, \mathrm{CDCl}_{3}\right)$ : 
$\delta$ 174.0, 64.1, 34.4, 31.8, 30.7, 29.2(2C), 29.1, 25.0, 22.6, 19.1, 14.1, 13.7, HRMS calcd for $\mathrm{C}_{13} \mathrm{H}_{26} \mathrm{O}_{2} 214.1933$; found 214.1934.

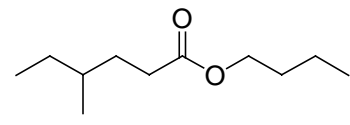

(3g) CAS registry No: $126391-29-5 .{ }^{1} \mathrm{H}$ NMR $\left(400 \mathrm{MHz}, \mathrm{CDCl}_{3}\right): \delta$ $4.03(\mathrm{t}, 2 \mathrm{H}, J=6.4 \mathrm{~Hz}), 2.28(\mathrm{~m}, 2 \mathrm{H}), 1.56(\mathrm{~m}, 2 \mathrm{H}), 1.37(\mathrm{~m}, 6 \mathrm{H}), 0.91(\mathrm{t}, 3 \mathrm{H}, J=6.8$ $\mathrm{Hz}), 0.84(\mathrm{~m}, 6 \mathrm{H}) .{ }^{13} \mathrm{C} \mathrm{NMR}\left(400 \mathrm{MHz}, \mathrm{CDCl}_{3}\right): \delta 174.3,64.1,33.9,32.2,31.5,30.7$, 29.1, 19.1, 18.8, 13.7, 11.2. HRMS calcd for $\mathrm{C}_{11} \mathrm{H}_{22} \mathrm{O}_{2}$ 186.1620; found 186.1624.

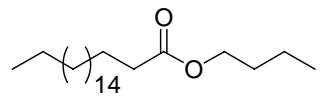

(3h) CAS registry No: $26718-87-6{ }^{1} \mathrm{H}$ NMR $\left(400 \mathrm{MHz}, \mathrm{CDCl}_{3}\right): \delta$ $4.04(\mathrm{t}, 2 \mathrm{H}, J=6.2 \mathrm{~Hz}), 2.26(\mathrm{t}, 2 \mathrm{H}, J=6.8 \mathrm{~Hz}), 1.59(\mathrm{~m}, 4 \mathrm{H}), 1.36(\mathrm{~m}, 2 \mathrm{H}), 1.26(\mathrm{~m}$, $30 \mathrm{H}), 0.91$ (t, $3 \mathrm{H}, J=6.8 \mathrm{~Hz}), 0.86$ (t, $3 \mathrm{H}, J=7.0 \mathrm{~Hz}) .{ }^{13} \mathrm{C} \mathrm{NMR}\left(400 \mathrm{MHz}, \mathrm{CDCl}_{3}\right): \delta$ 174.0, 64.1, 34.4, 32.1, 31.9, 30.7, 29.7(3C), 29.5, 29.4, 29.3, 29.2, 27.2, 25.0, 22.7, 19.1, $14.1,13.7$

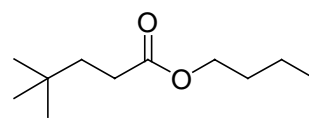

(3i) ${ }^{1} \mathrm{H}$ NMR $\left(400 \mathrm{MHz}, \mathrm{CDCl}_{3}\right): \delta 4.04(\mathrm{t}, 2 \mathrm{H}, J=6.5), 2.25(\mathrm{t}, 2 \mathrm{H}, J$ $=6.2 \mathrm{~Hz}), 1.45-1.51(\mathrm{~m}, 4 \mathrm{H}), 1.31-1.38(\mathrm{~m}, 2 \mathrm{H}), 0.91(\mathrm{t}, 3 \mathrm{H}, J=7.2 \mathrm{~Hz}), 0.86(\mathrm{~s}, 9 \mathrm{H})$. ${ }^{13} \mathrm{C}$ NMR (400 MHz, $\mathrm{CDCl}_{3}$ ): $\delta 174.6,64.2,38.6,30.7,30.1,30.0,28.9(3 \mathrm{C}), 19.1,13.7$. HRMS calcd for $\mathrm{C}_{11} \mathrm{H}_{26} \mathrm{O}_{2}$ 186.1620; found 186.1622.

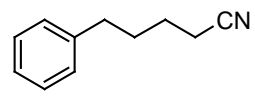

(3j) CAS registry No: 7726-45-6. ${ }^{1} \mathrm{H}$ NMR $\left(400 \mathrm{MHz}, \mathrm{CDCl}_{3}\right): \delta 7.26-$ $7.30(\mathrm{~m}, 2 \mathrm{H}), 7.15-7.21(\mathrm{~m}, 3 \mathrm{H}), 2.65(\mathrm{t}, 2 \mathrm{H}, J=7.0 \mathrm{~Hz}), 2.33(\mathrm{t}, 2 \mathrm{H}, J=7.2 \mathrm{~Hz}), 1.76$ (m, 2H), 1.65 (m, 2H). ${ }^{13} \mathrm{C} \mathrm{NMR} \mathrm{(400} \mathrm{MHz,} \mathrm{CDCl}_{3}$ ): $\delta$ 141.2, 128.4, 128.3, 126.0, 34.9, 30.2, 24.8, 17.0. HRMS calcd for $\mathrm{C}_{11} \mathrm{H}_{13} \mathrm{~N}$ 159.1048; found 159.1048. 


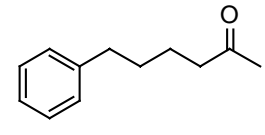

(3k) CAS registry No: $14171-89-2 .{ }^{1} \mathrm{H}$ NMR $\left(400 \mathrm{MHz}, \mathrm{CDCl}_{3}\right): \delta 7.26$ (m, 2H), 7.14-7.18 (m, 3H), $2.60(\mathrm{t}, 2 \mathrm{H}, J=6.8 \mathrm{~Hz}), 2.43(\mathrm{t}, 2 \mathrm{H}, J=6.4 \mathrm{~Hz}), 2.1(\mathrm{~s}, 3 \mathrm{H})$, 1.57-1.62 (m, 4H). ${ }^{13} \mathrm{C}$ NMR (400 MHz, $\left.\mathrm{CDCl}_{3}\right): \delta 217.0,142.2,128.4,128.3,125.8$, 43.6, 35.7, 30.9, 29.8, 23.5.

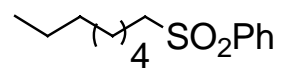

(3I) CAS registry No: 34009-05-7. ${ }^{1} \mathrm{H}$ NMR $\left(400 \mathrm{MHz}, \mathrm{CDCl}_{3}\right): \delta 7.88$ (m, 2H, ), $7.63(\mathrm{~m}, 1 \mathrm{H}), 7.55(\mathrm{~m}, 2 \mathrm{H}), 3.05(\mathrm{t}, 2 \mathrm{H}, J=6.4 \mathrm{~Hz}), 1.63-1.69(\mathrm{~m}, 2 \mathrm{H}), 1.28-$ $1.33(\mathrm{~m}, 2 \mathrm{H}), 1.19-1.23(\mathrm{~m}, 10 \mathrm{H}), 0.83(\mathrm{t}, 3 \mathrm{H}, J=6.8 \mathrm{~Hz}) .{ }^{13} \mathrm{C} \mathrm{NMR}\left(400 \mathrm{MHz}, \mathrm{CDCl}_{3}\right)$ : $\delta$ 139.3, 133.6, 129.2, 128.0, 56.3, 31.6, 28.9, 28.8, 28.2, 22.6, 22.5, 13.9. HRMS calcd for $\mathrm{C}_{11} \mathrm{H}_{16} \mathrm{O}_{2} \mathrm{~S} 212.0871$; found 212.0875 .

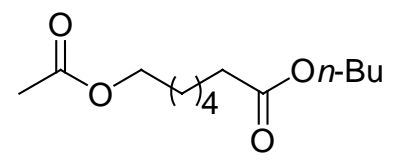

(3m) ${ }^{1} \mathrm{H}$ NMR (400 MHz, $\left.\mathrm{CDCl}_{3}\right): \delta 4.03(\mathrm{~m}, 4 \mathrm{H}), 2.26(\mathrm{t}, 3 \mathrm{H}, J$ $=6.4 \mathrm{~Hz}), 2.01(\mathrm{~s}, 3 \mathrm{H}), 1.50-1.59(\mathrm{~m}, 6 \mathrm{H}), 1.32-1.39(\mathrm{~m}, 6 \mathrm{H}), 0.89(\mathrm{t}, 3 \mathrm{H}, J=7.0 \mathrm{~Hz})$. ${ }^{13} \mathrm{C}$ NMR (400 MHz, $\mathrm{CDCl}_{3}$ ): $\delta 173.7,171.1,64.4,64.1,34.2,30.7,28.7,28.4,25.6$, 24.8, 20.9, 19.1, 13.6. HRMS calcd for $\mathrm{C}_{10} \mathrm{H}_{18} \mathrm{O}_{4}$ 202.1205; found 202.1207. 


\section{References:}

1. (a) Toda, S.; Miyamoto, M.; Kinoshita, H.; Inomata, K. Bull. Chem. Soc. Jpn. 1991, 64, 3600.

2. (a) Bureau, N.; Defiolle, D.; de Hemptinne, J. -C. Fluid Phase Equilibria 2002, 194-197, 831-846.

(b) Marosi, L.; Schlenk, W. Jr. Justus Liebigs Annalen der Chemie 1973, 4, 584-598.

3. (a) Baban, J. A.; Roberts, B. P.; Ingold, C. J. Chem Soc, Perkin Trans. 2 1988, 1195-1200. (b) Tomonori, Y; Hideo, T. Synlett 2004, 9, 1604-1606.

4. (a) Huo, S. Org. Lett. 2003, 5, 423-425. (b) Wipf, P.; Venkataraman,S. J. Org. Chem. 1993, 58, 3455-3458.

5. (a) Donkor, I. O.; Li, H.; Queener, S. F. Eur. J. Med. Chem. 2003, 38, 605-611. (b) Tan, Z.; Qu, Z.; Chen, B.; Wang, J. Tetrahedron 2000, 56, 7457-7461.

6. (a) Heinsman, N. W. J. T.; Orrenius, S. C.; Marcelis, C. L. M.; De Sousa Teixeira, A.; Franssen, M. C. R.; Van Der Padt, A.; Jongejan, J. A.; De Groot, Ae. Biocatalysis and Biotransformation 1998, 16, 145-162. (b) Kim, H. J.; Lindsay, R. C. J. Food Composition and Analysis 1989, 2, 118 131. (c) Vasi, I. G.; Nanavati, N. T. J. Inst. Chemists (India) 1976, 48, Pt. 4, 198-201.

7. (a) Hu, Y.; Chen, Z. -C.; Le, Z. -G; Zheng, Q. -G. Synth. Commun. 2004, 34, 4031-4035. (b) King, J. F.; Rathore, R.; Guo, Z.; Li, M.; Payne, N. C. J. Am. Chem. Soc. 2000, 122, 10308-10324. (c) Russell, G. A.; Shi, B. Z.; Jiang, W.; Hu, S.; Kim, B. H.; Baik, W. J. Am. Chem. Soc. 1995, 117, 3952-3962.

8. Hauptmann, S.; Brandes, F.; Brauer, E.; Gabler, W. Journal fuer Praktische Chemie (Leipzig) 1964, 25, 56-68.

9. Aono, M.; Terao, Y.; Achiwa, K. Heterocycles 1995, 40, 249-260. 


\section{${ }^{1} \mathrm{H}$ NMR spectrum of $3 \mathbf{a}$}

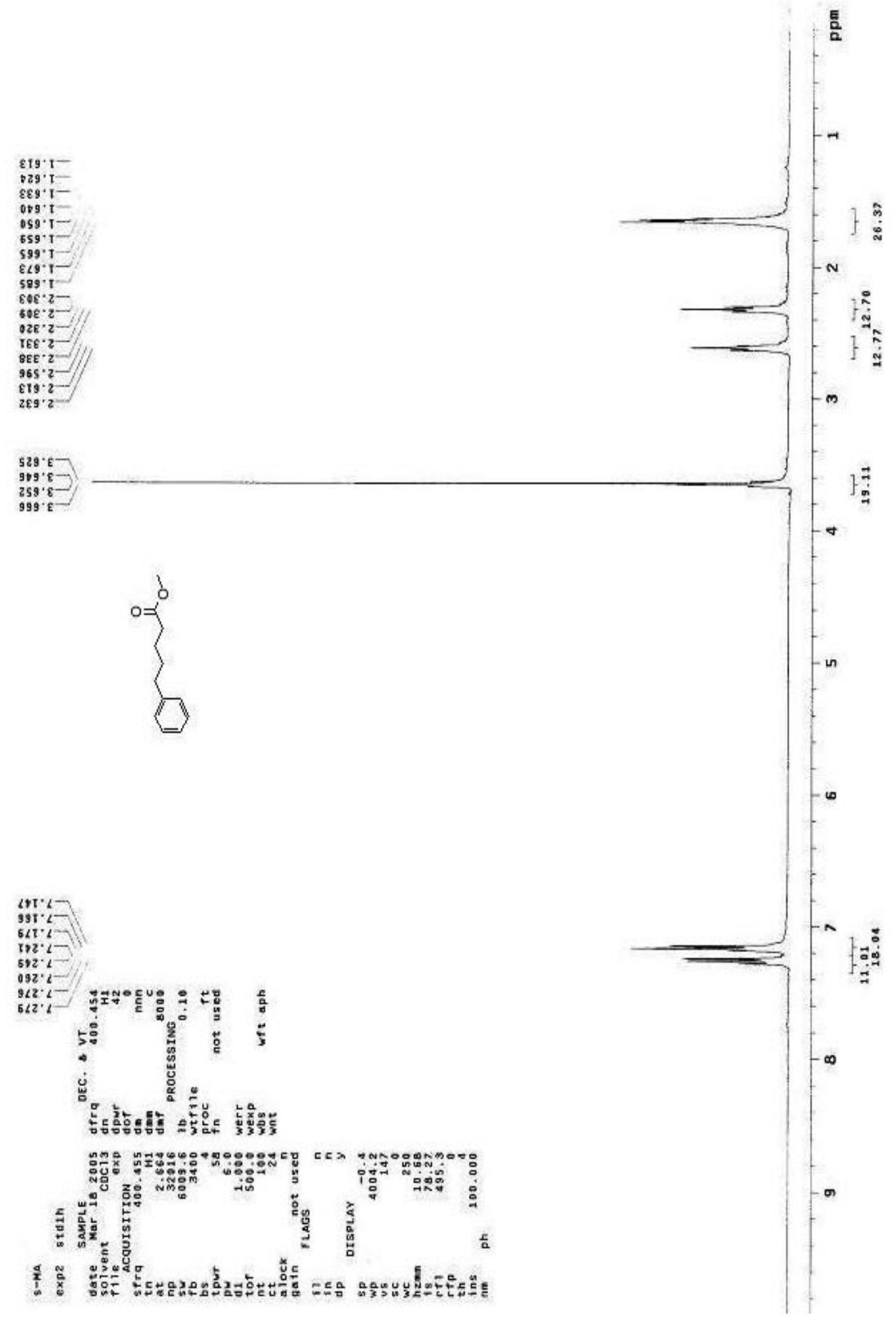




\section{${ }^{13} \mathrm{C}$ NMR spectrum of $3 \mathrm{a}$}
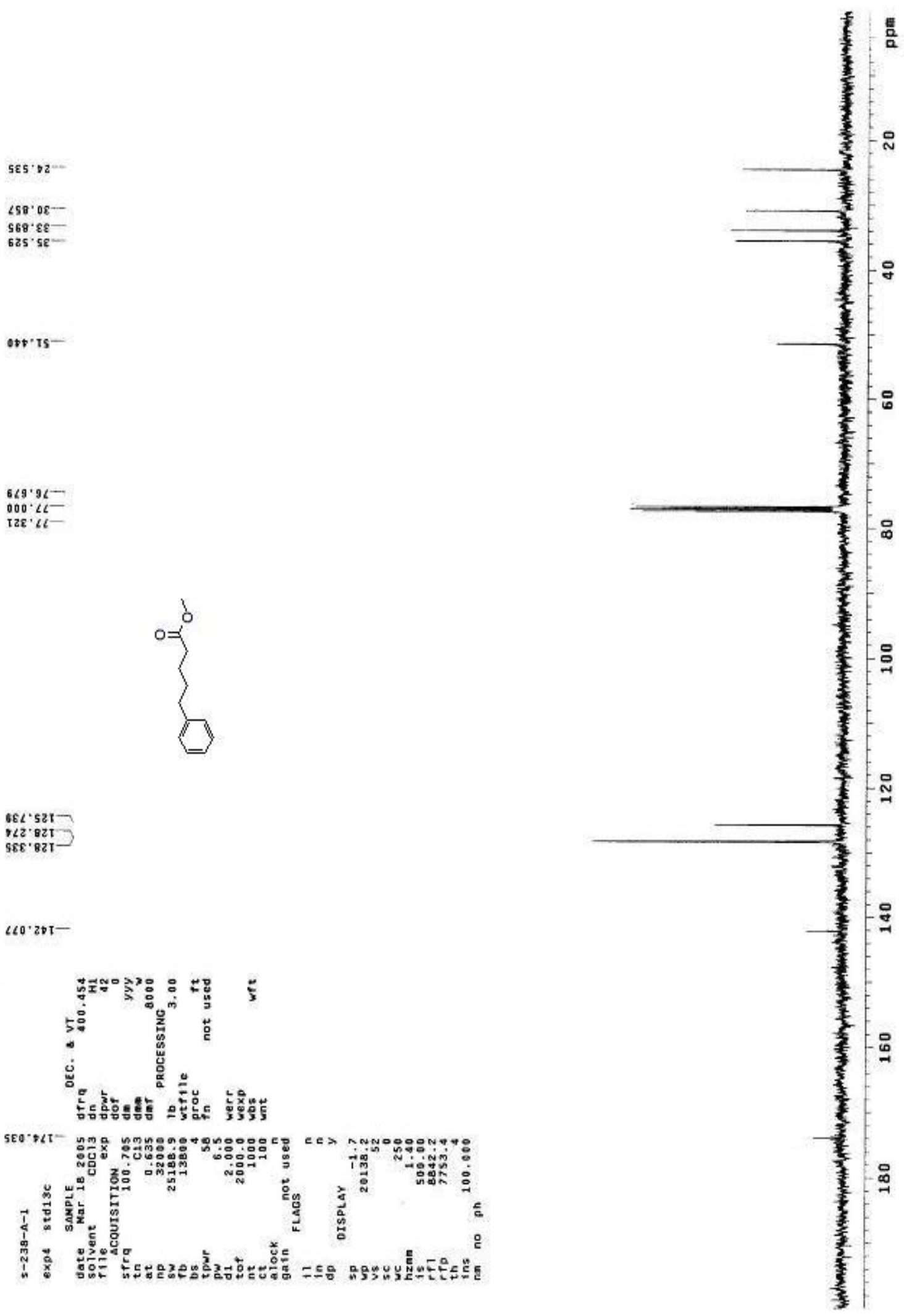
${ }^{1} \mathrm{H}$ NMR spectrum of $\mathbf{3 b}$

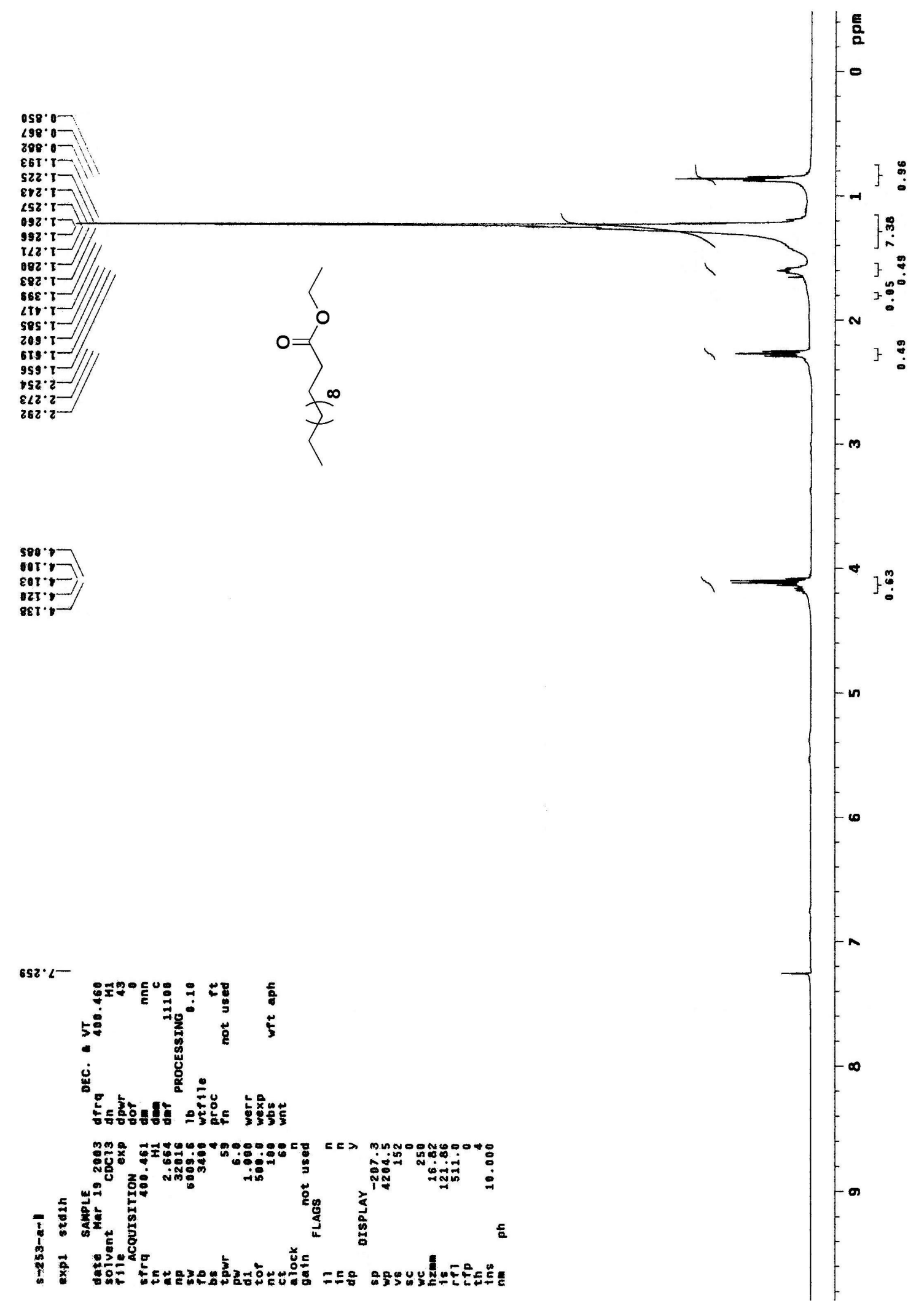


${ }^{13} \mathrm{C}$ NMR spectrum of $3 \mathrm{~b}$
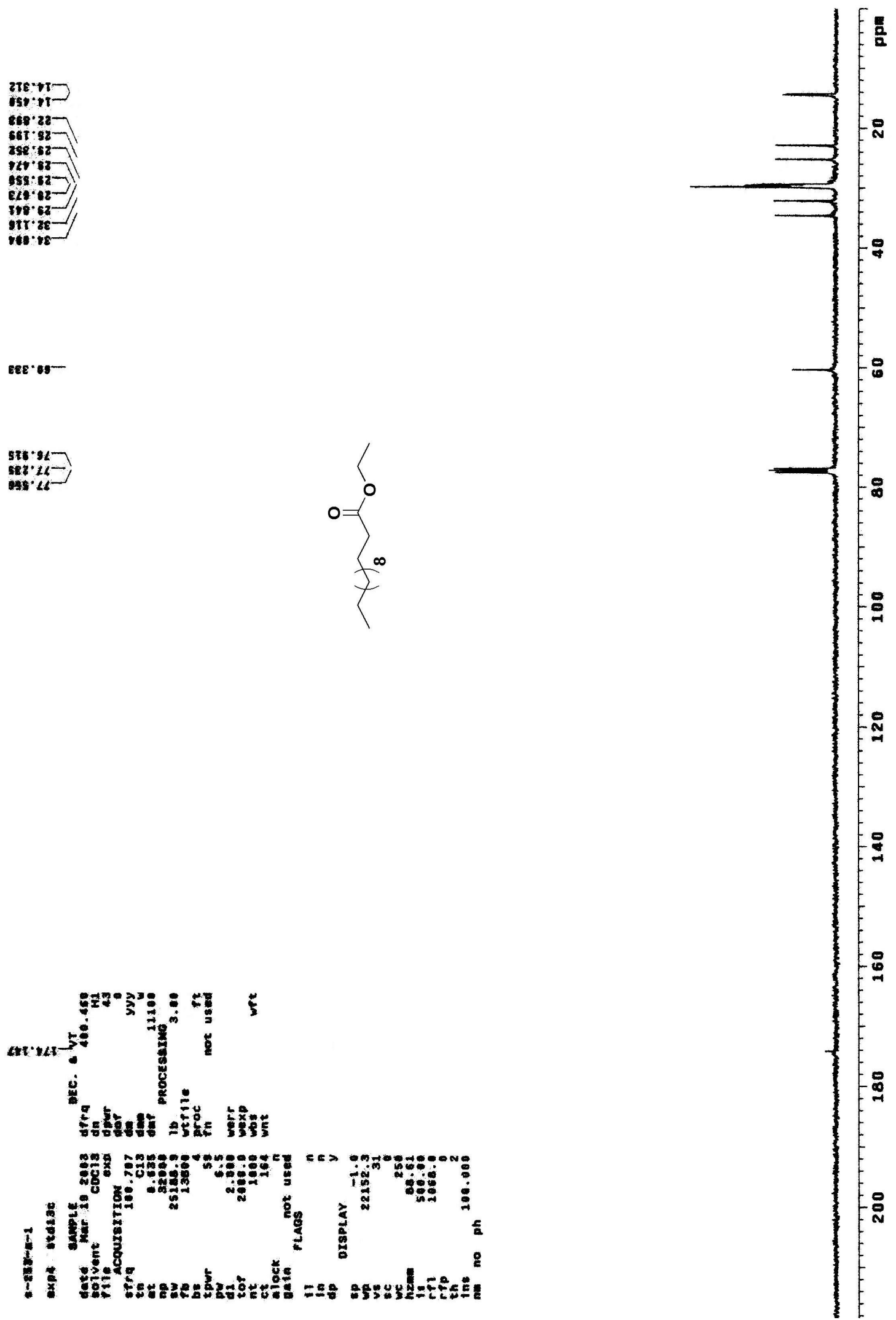


\section{${ }^{\text {I}} \mathrm{H}$ NMR spectrum of $3 \mathrm{c}$}

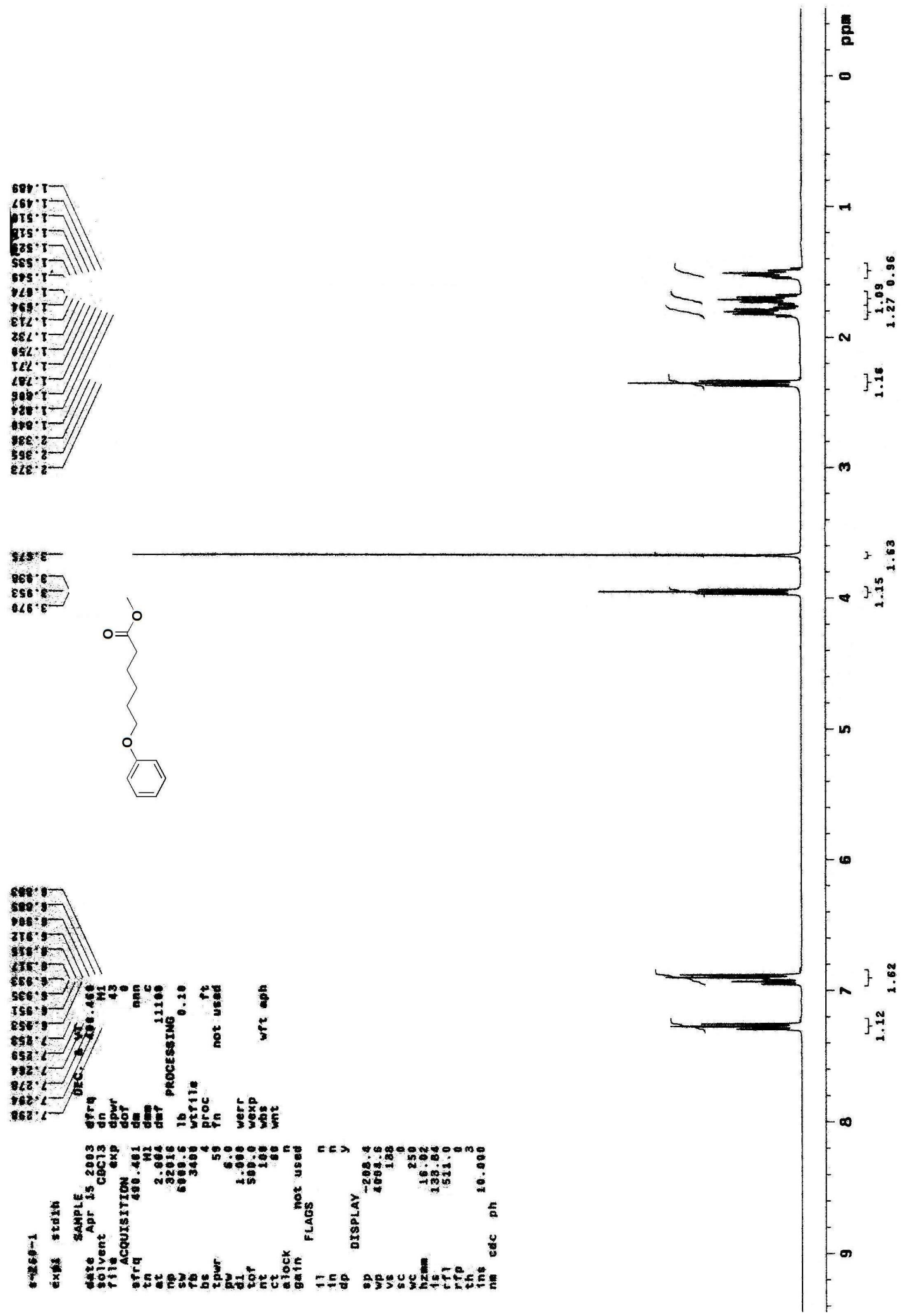




\section{${ }^{13} \mathrm{C}$ NMR spectrum of $3 \mathrm{c}$}
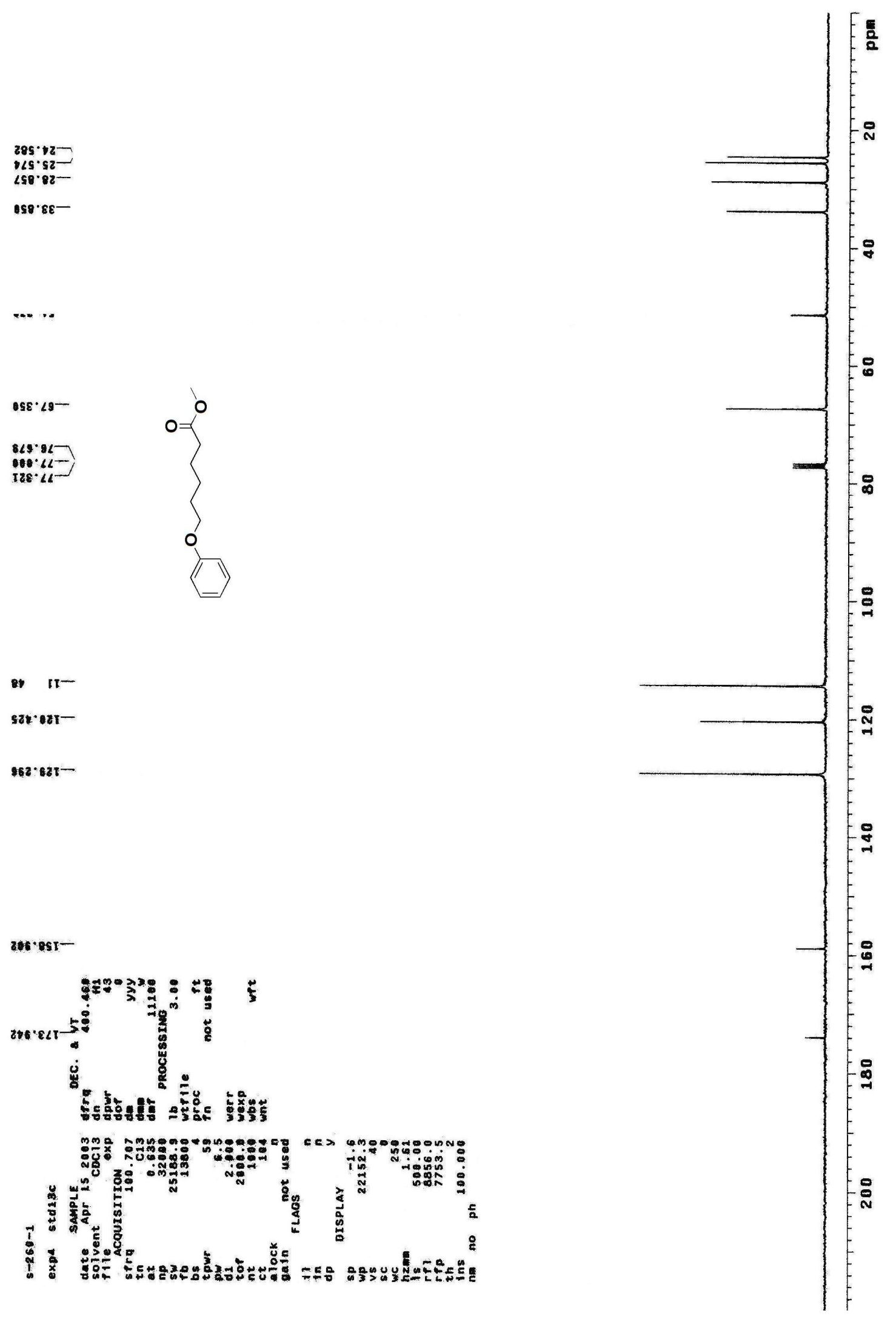
${ }^{1} \mathrm{H}$ NMR spectrum of $\mathbf{3 d}$
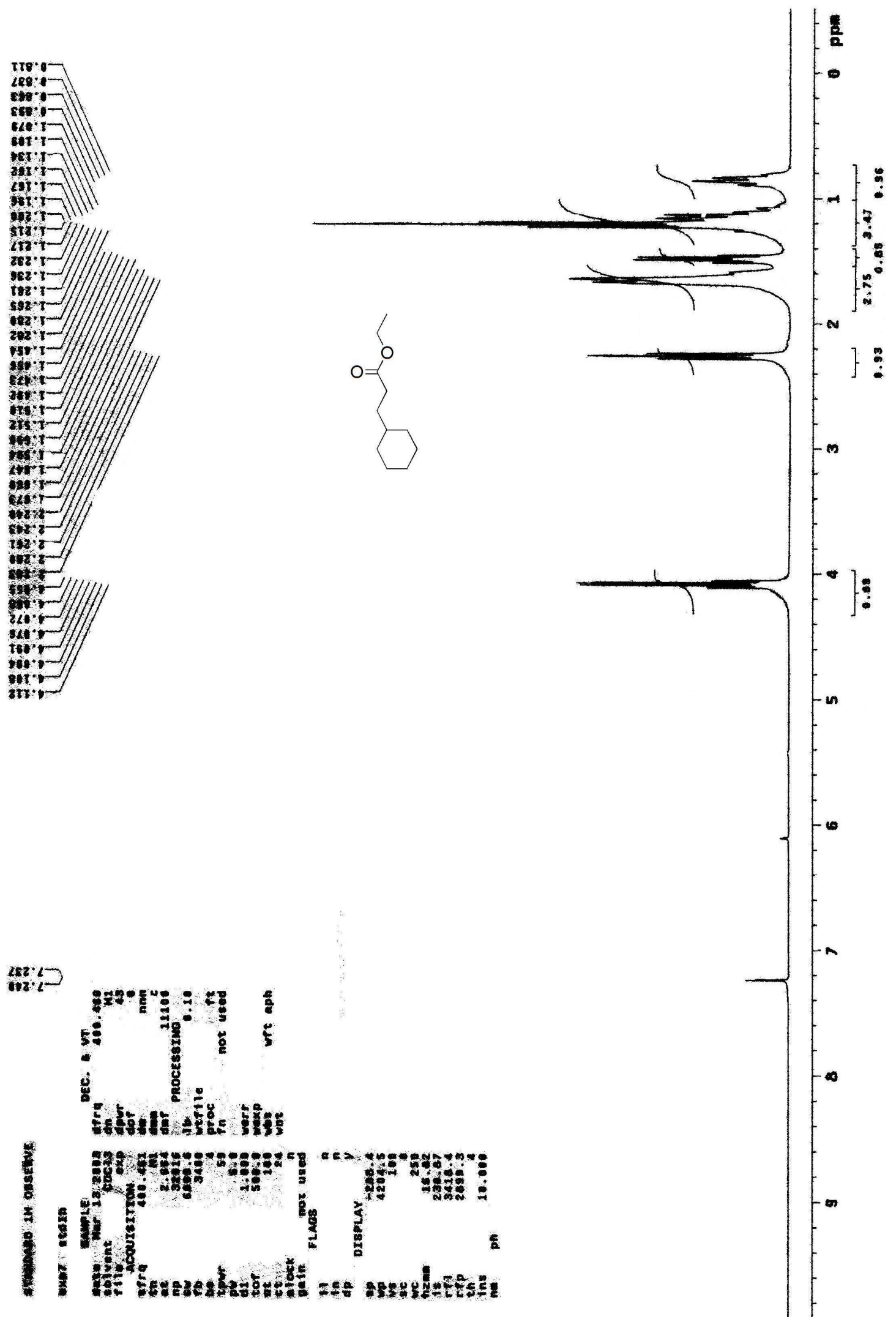
${ }^{13} \mathrm{C}$ NMR spectrum of $3 \mathrm{~d}$

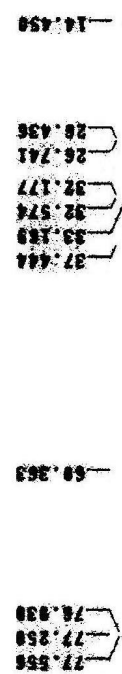

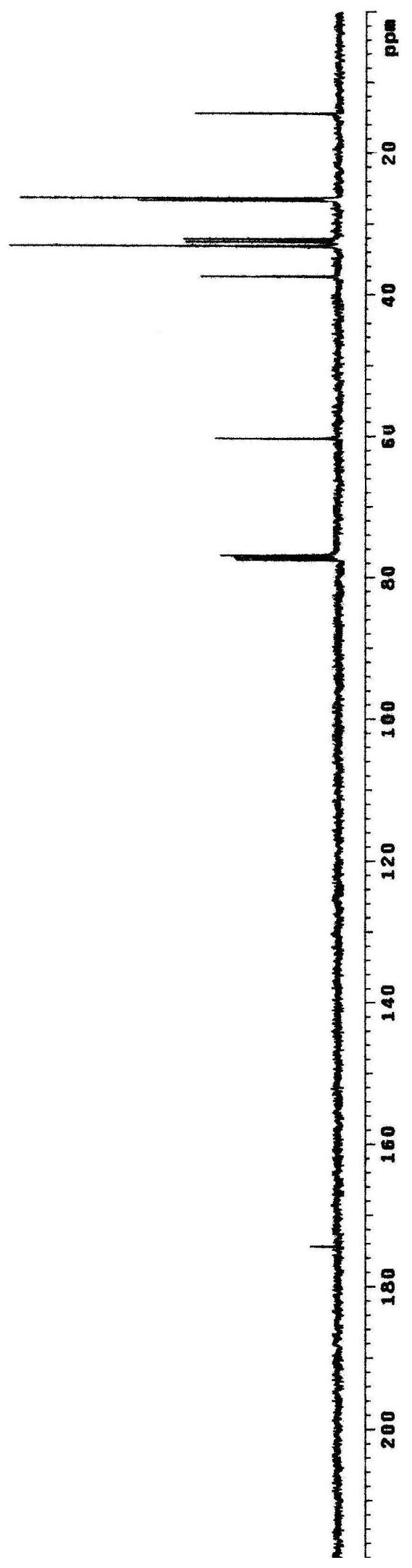




\section{${ }^{1} \mathrm{H}$ NMR spectrum of $3 \mathrm{e}$}

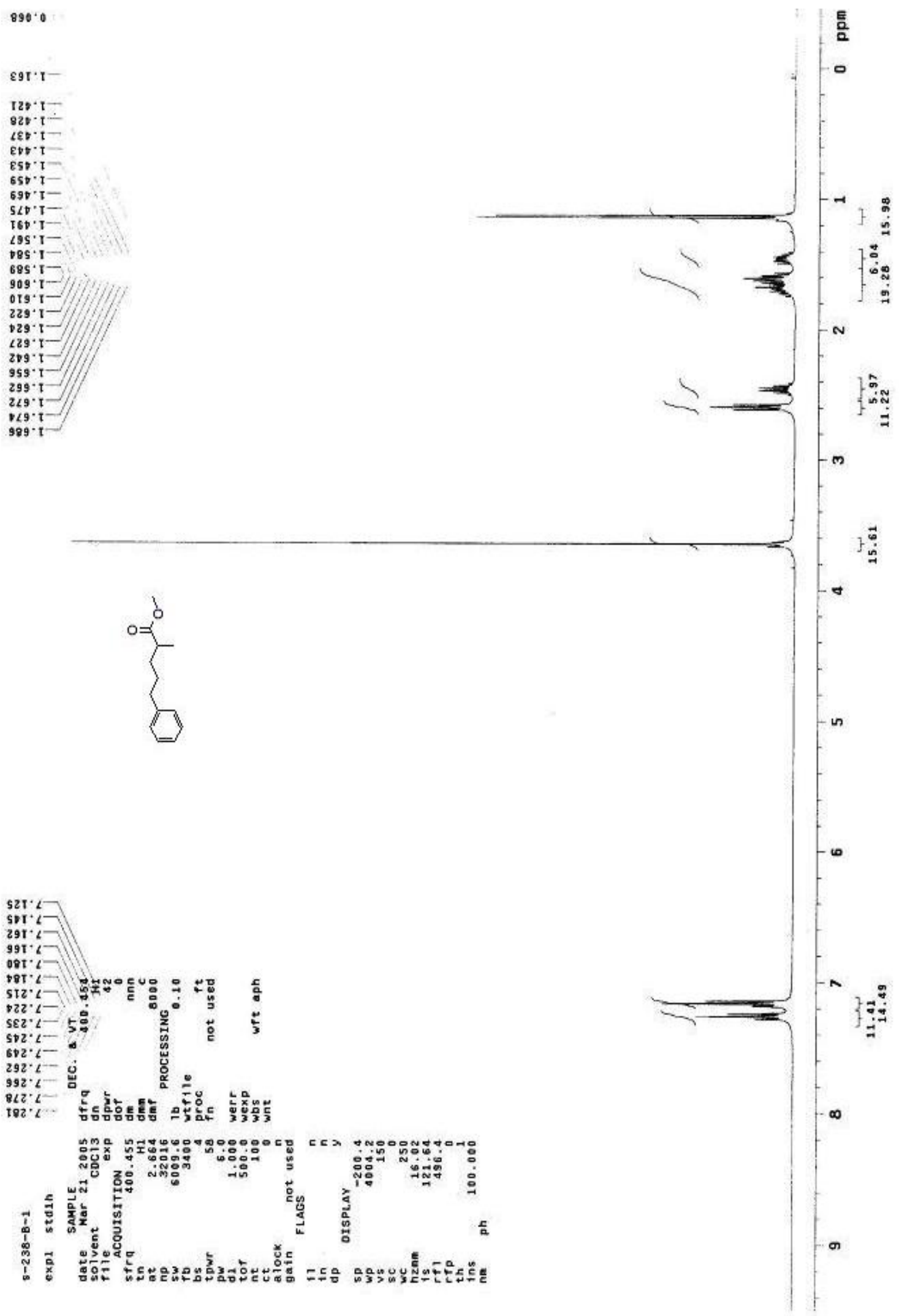




\section{${ }^{13} \mathrm{C}$ NMR and DEPT spectrum of $3 \mathrm{e}$}

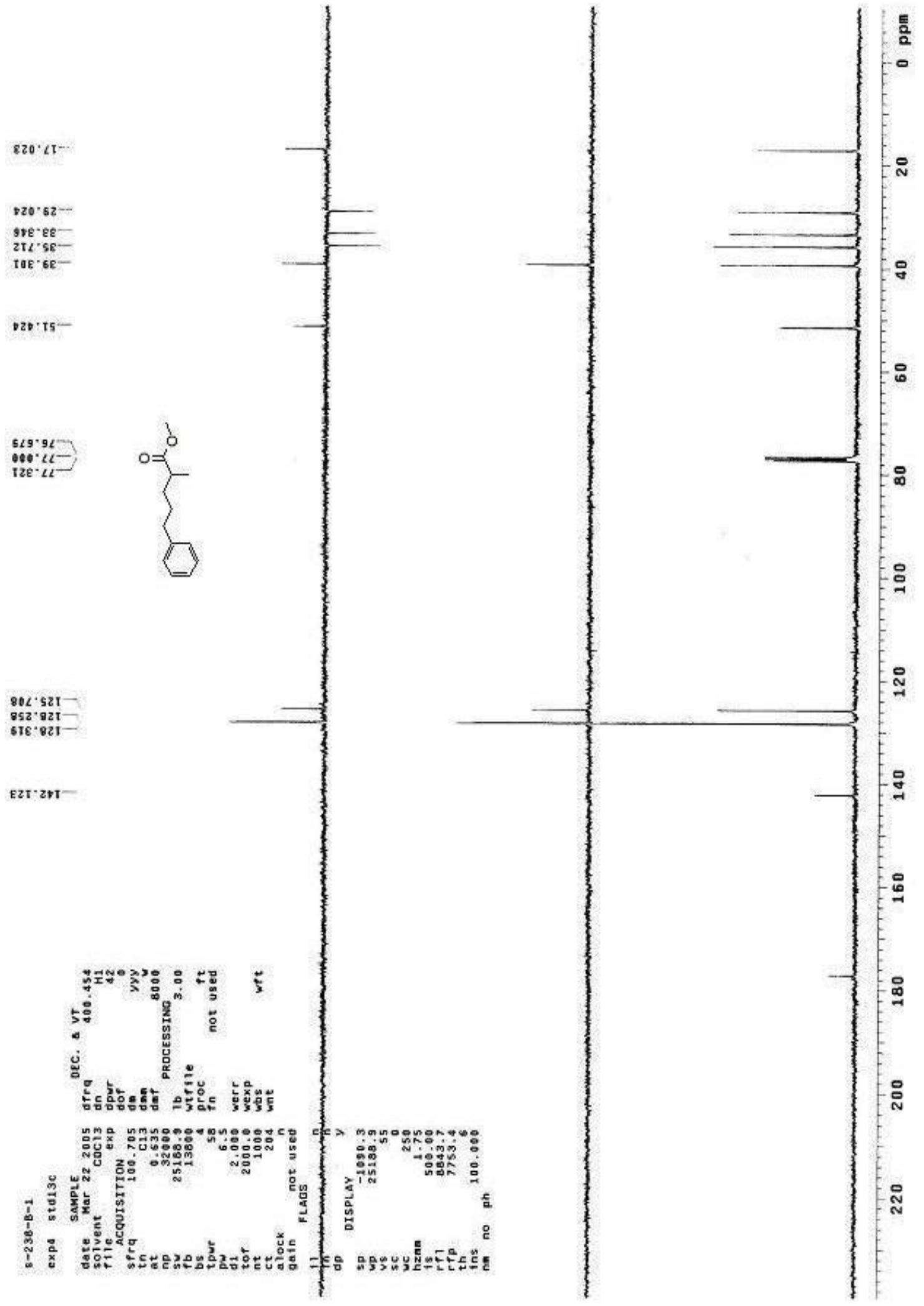




\section{${ }^{\text {I}} \mathrm{H}$ NMR spectrum of $3 \mathrm{f}$}

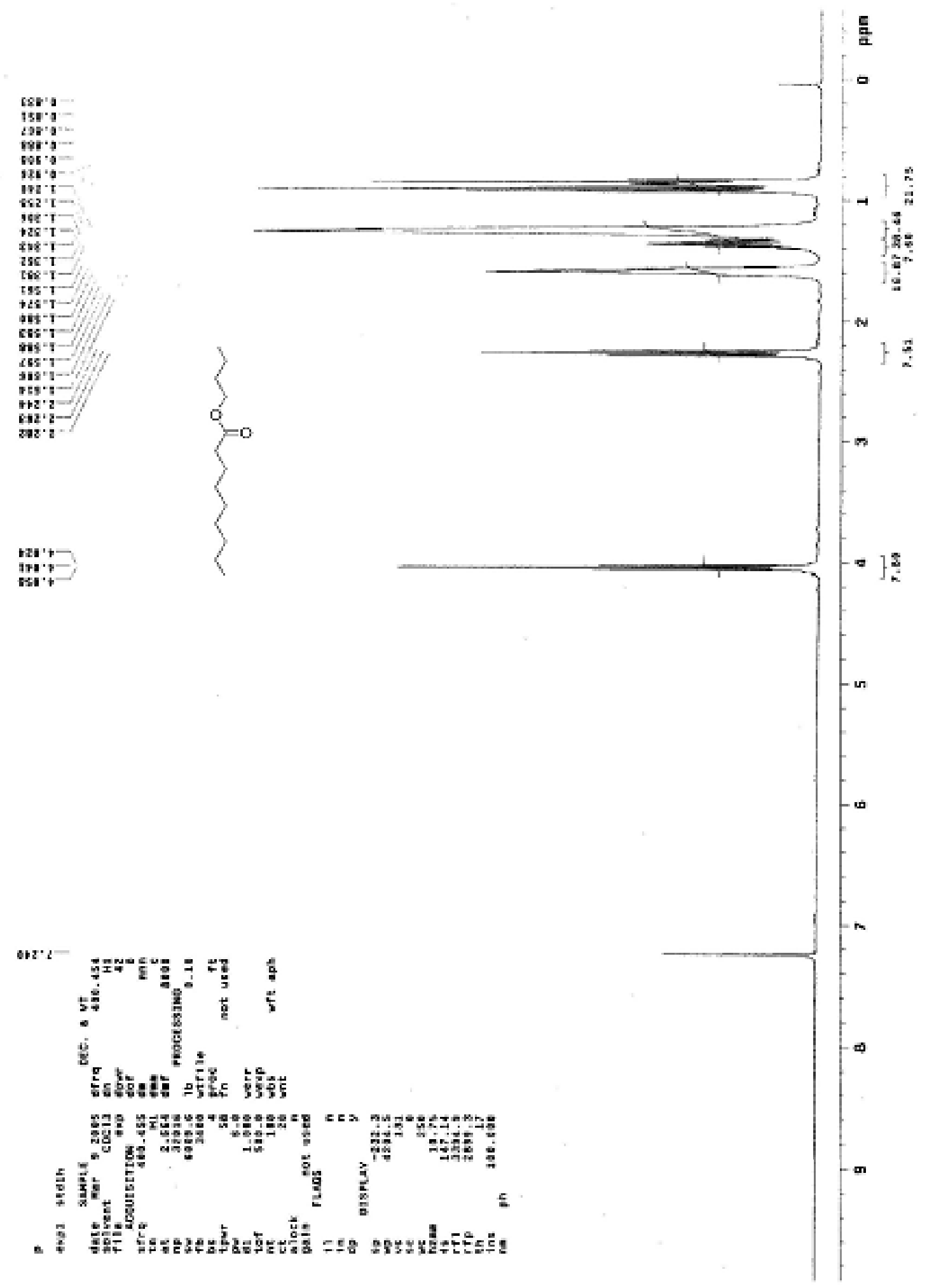




\section{${ }^{13} \mathrm{C}$ NMR spectrum of $3 \mathrm{f}$}

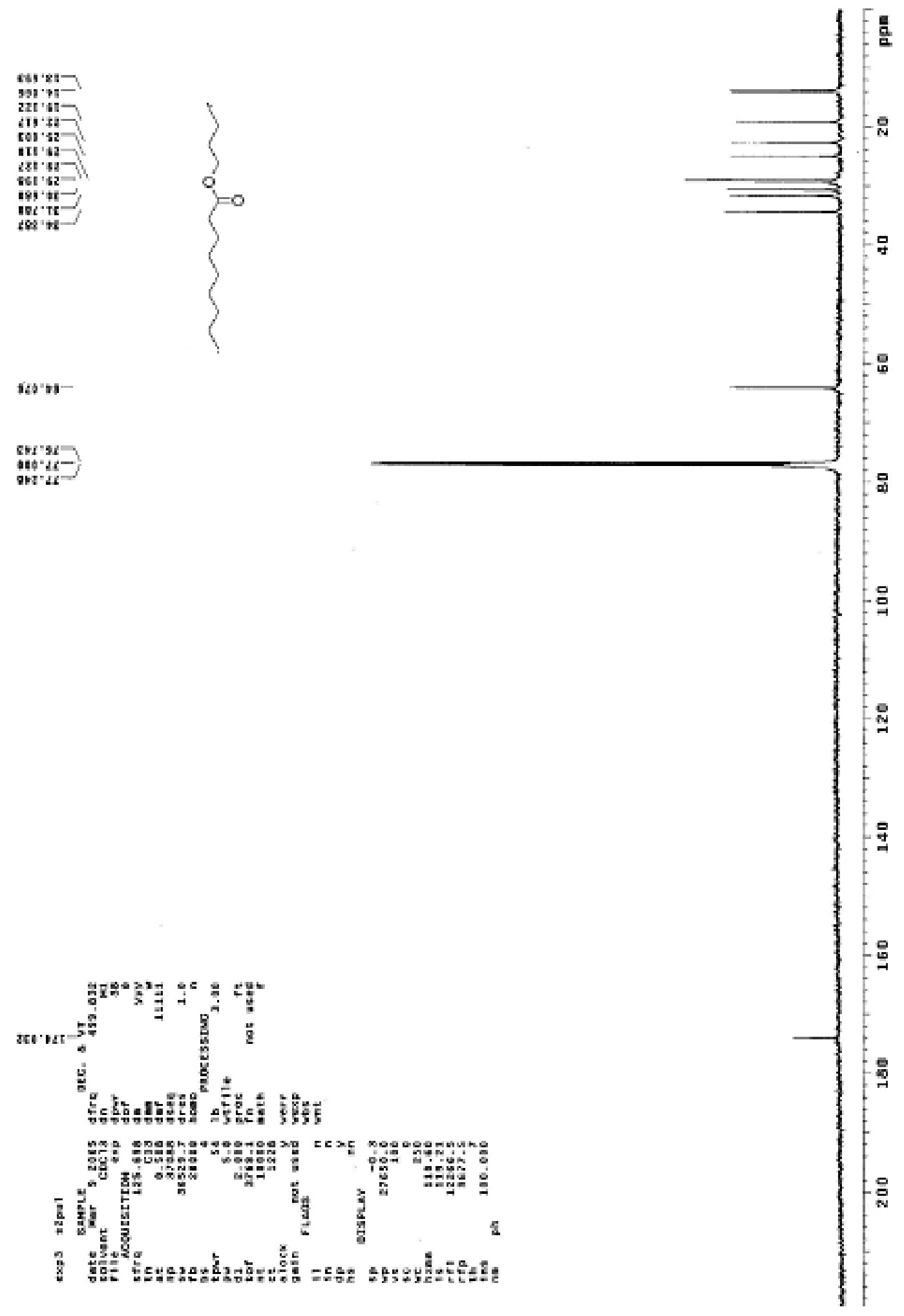




\section{${ }^{\mathrm{I}} \mathrm{H}$ NMR and DEPT spectrum of $3 \mathrm{~g}$}

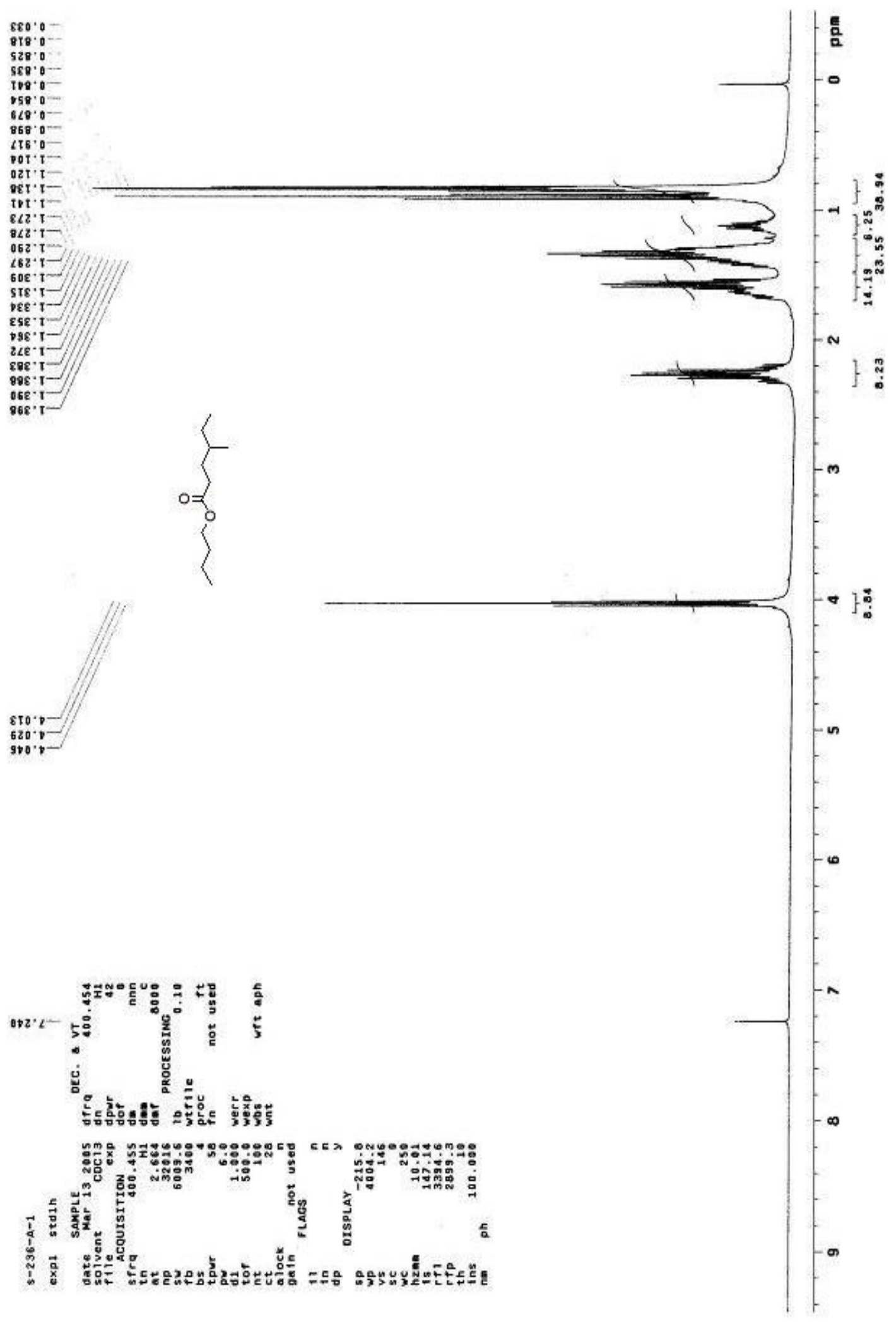




\section{${ }^{13} \mathrm{C}$ NMR and DEPT spectrum of $3 \mathrm{~g}$}
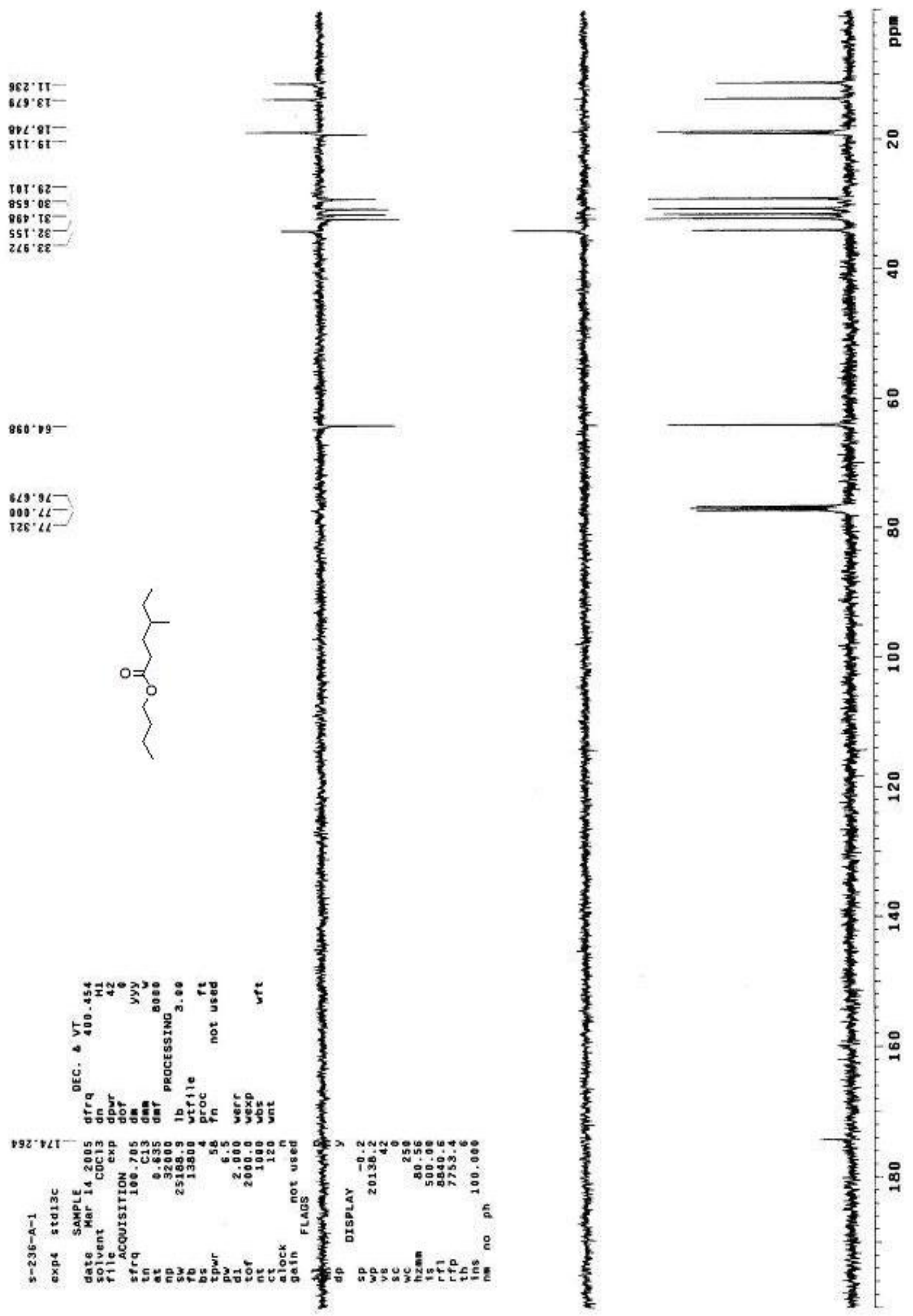


\section{${ }^{1} \mathrm{H}$ NMR spectrum of $3 \mathrm{~h}$}

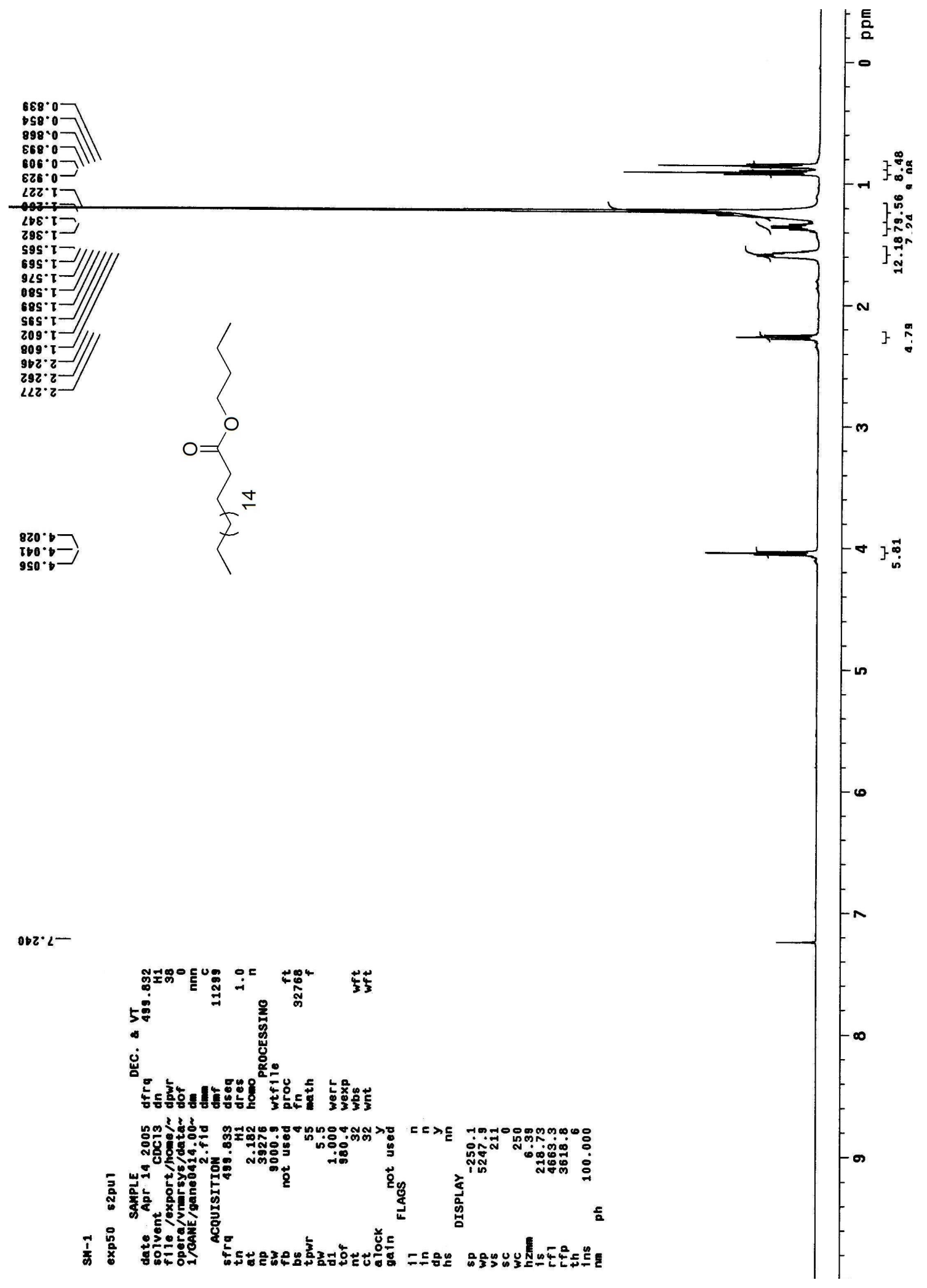




\section{${ }^{13} \mathrm{C}$ NMR spectrum of $3 \mathrm{~h}$}

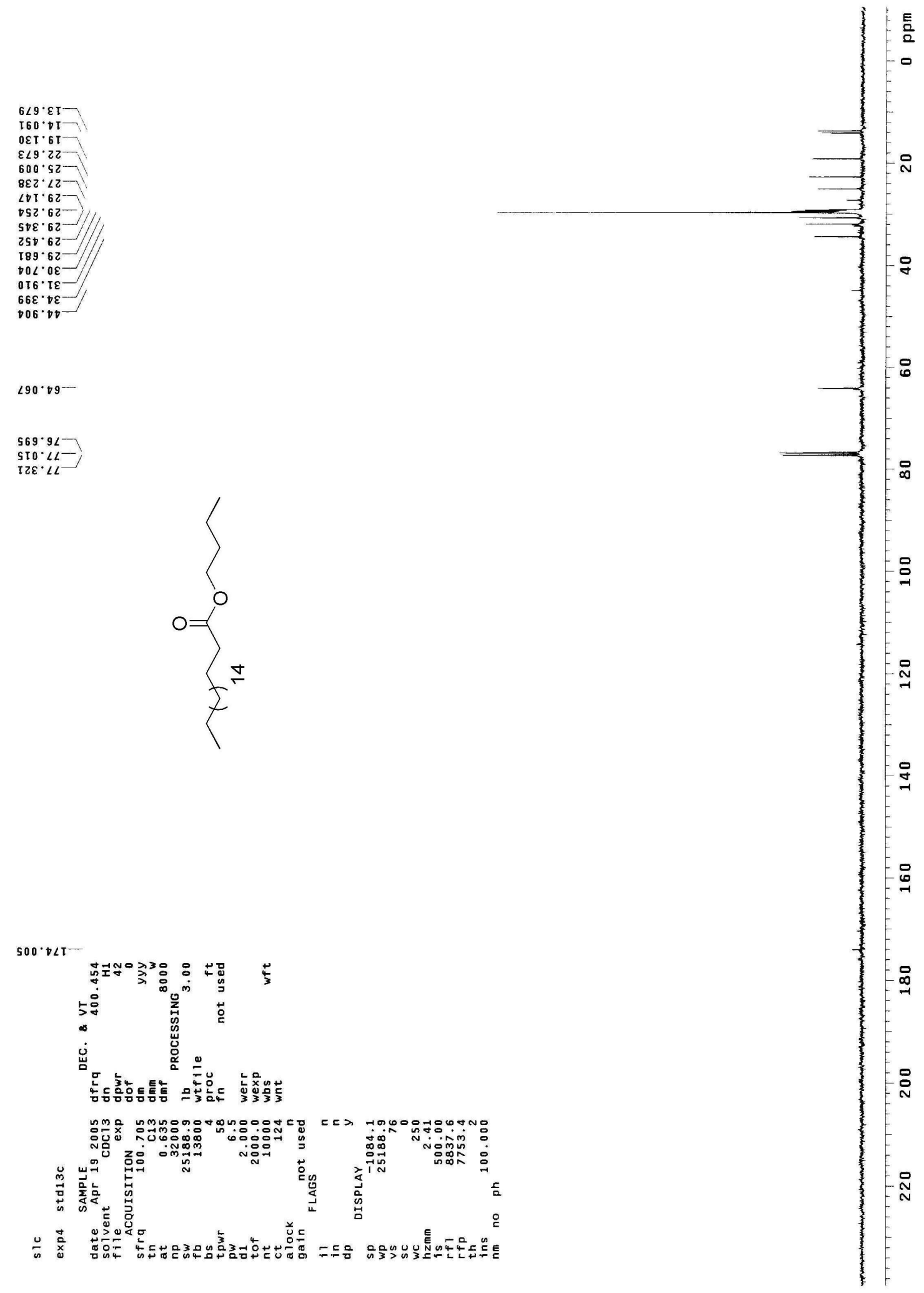




\section{${ }^{\text {I}} \mathrm{H}$ NMR spectrum of $3 \mathrm{i}$}

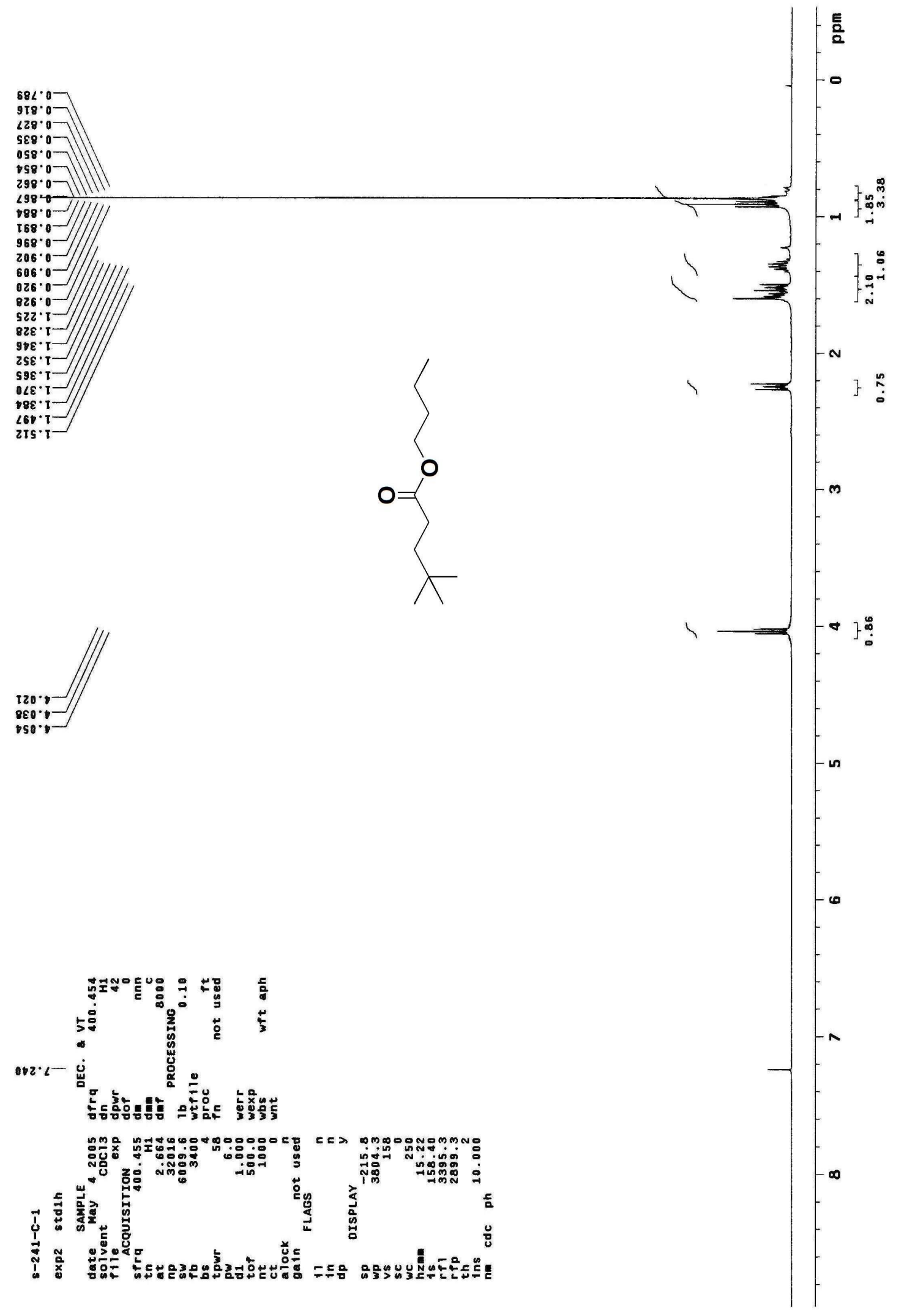




\section{${ }^{13} \mathrm{C}$ NMR spectrum of $3 \mathrm{i}$}

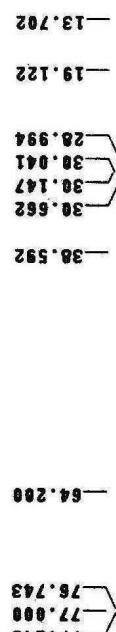

800.12

$907 \cdot 26$
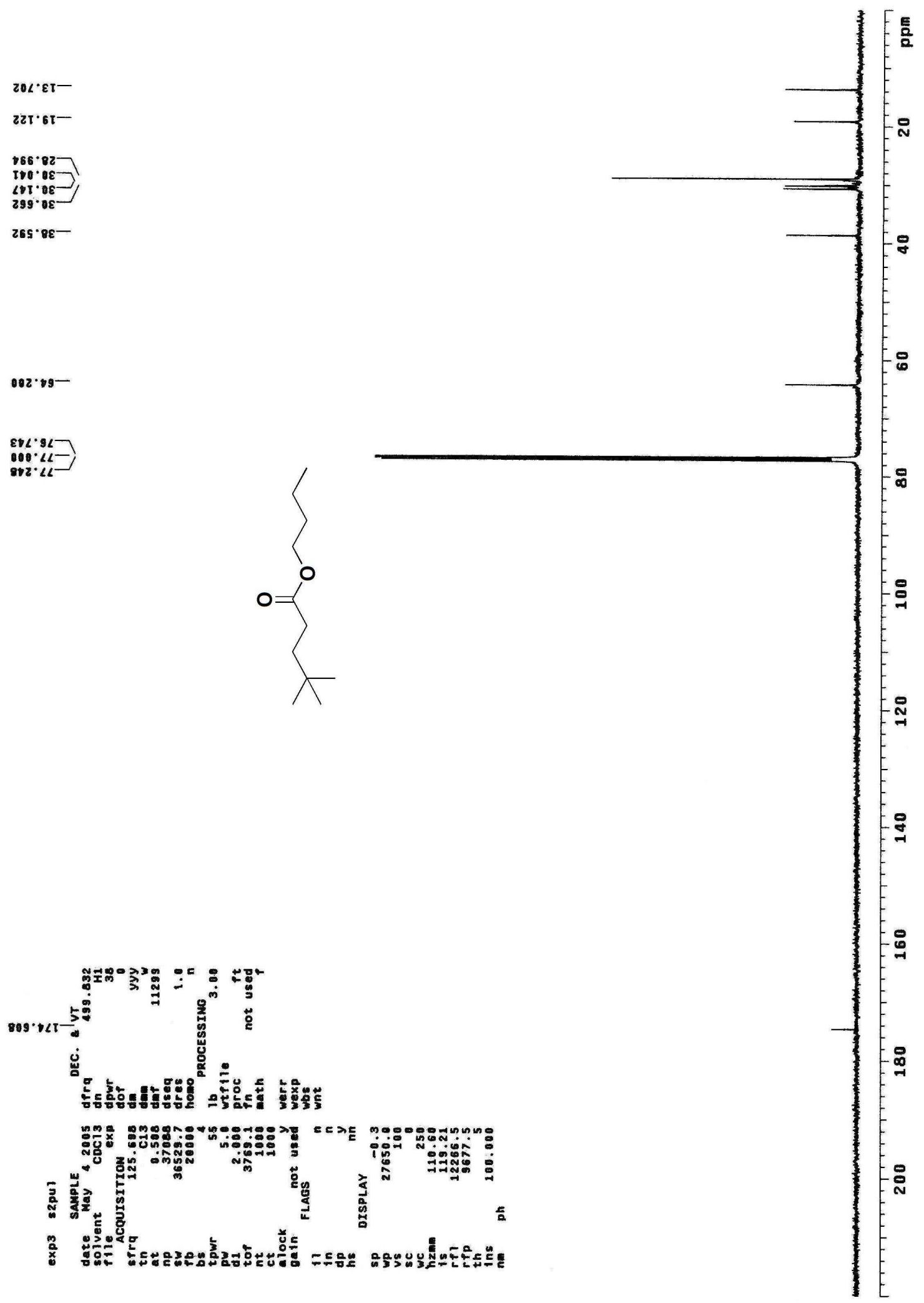

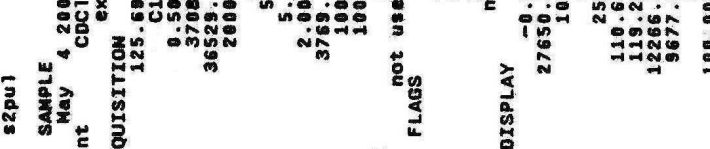

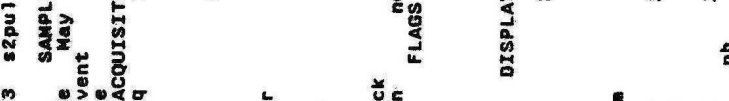

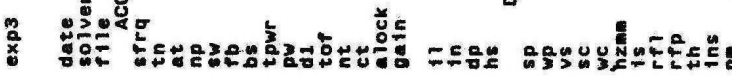


${ }^{1} \mathbf{H}$ NMR spectrum of $\mathbf{3 j}$

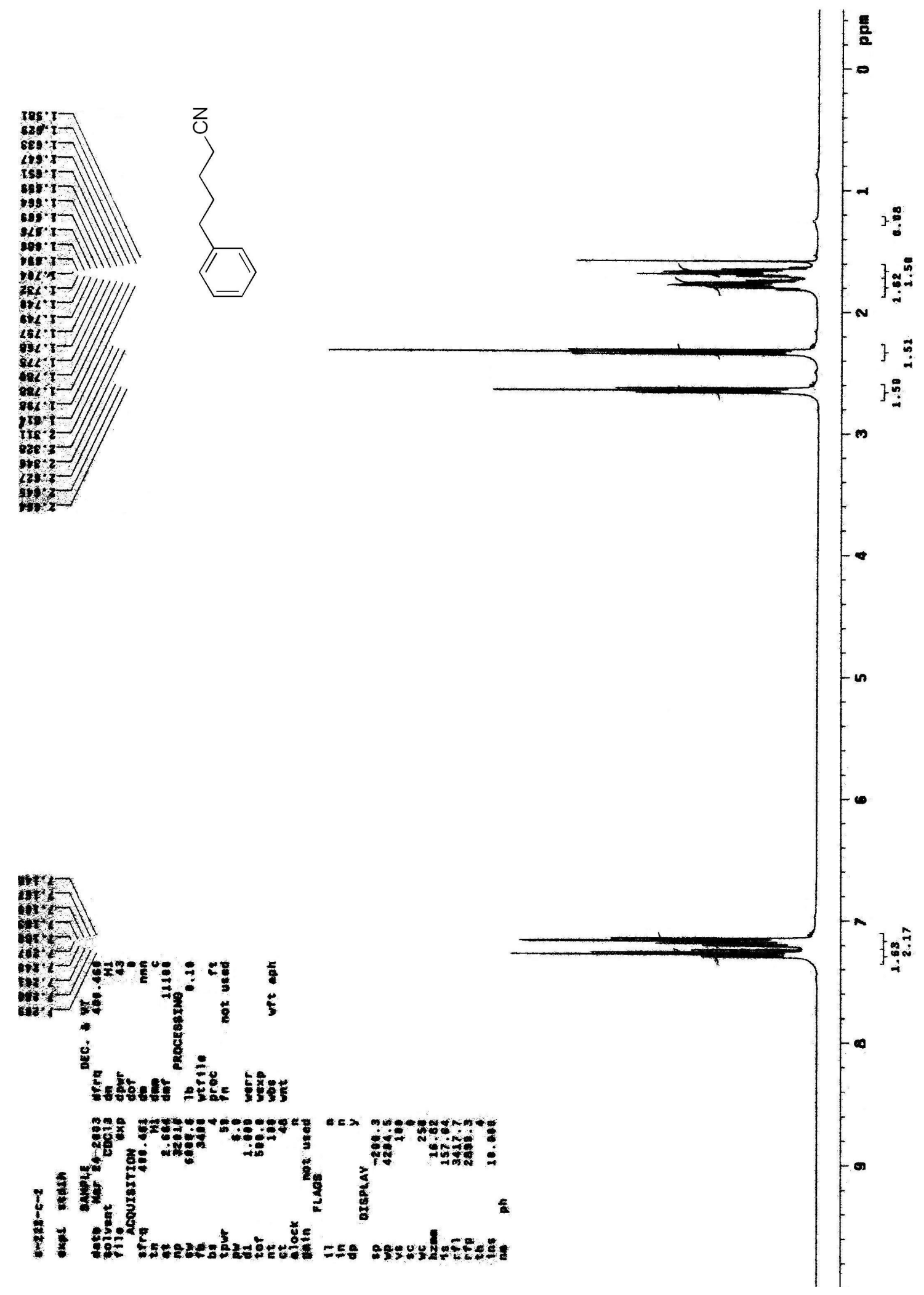


${ }^{13} \mathrm{C}$ NMR spectrum of $3 \mathrm{j}$
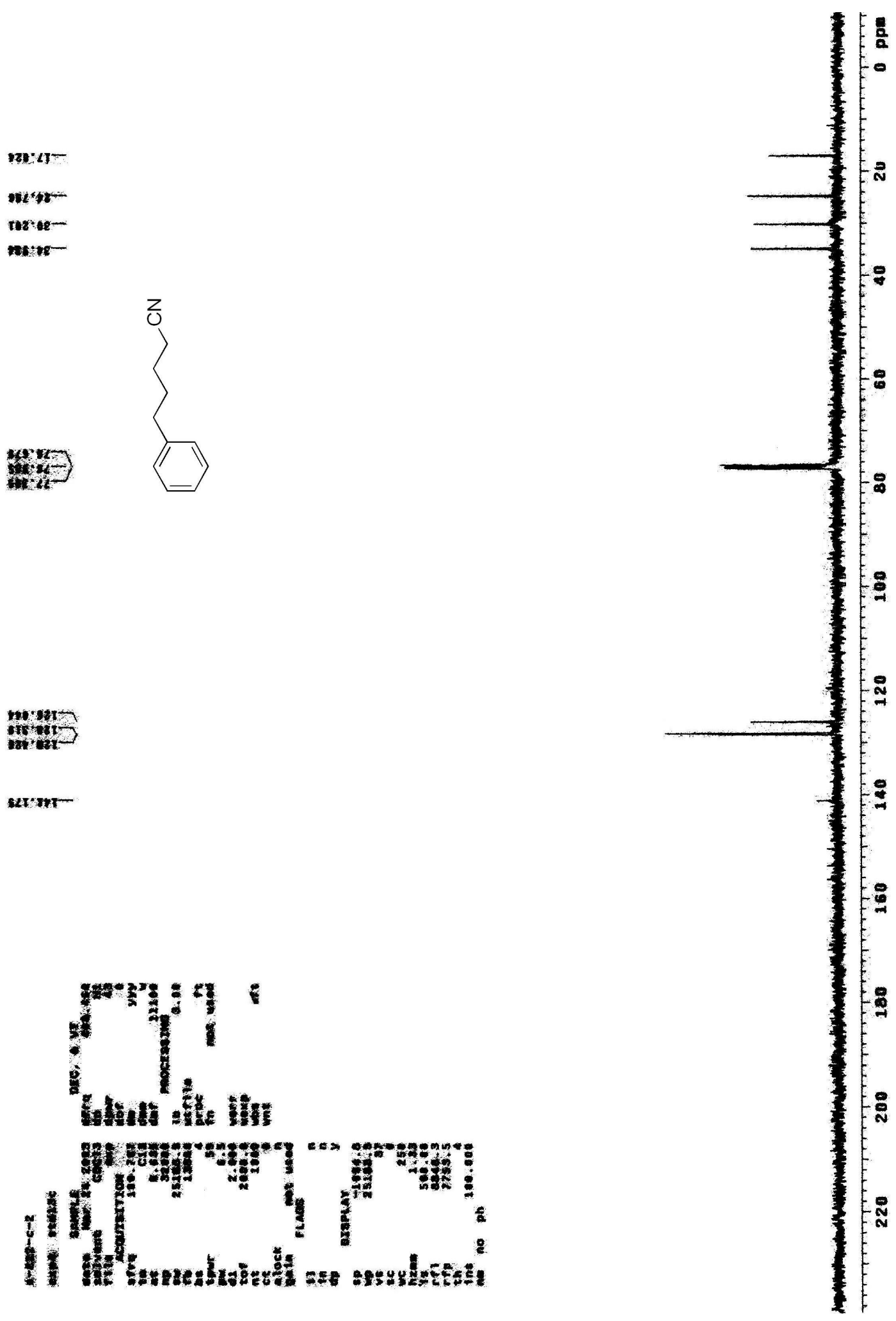
${ }^{1} \mathbf{H}$ NMR spectrum of $3 \mathbf{k}$

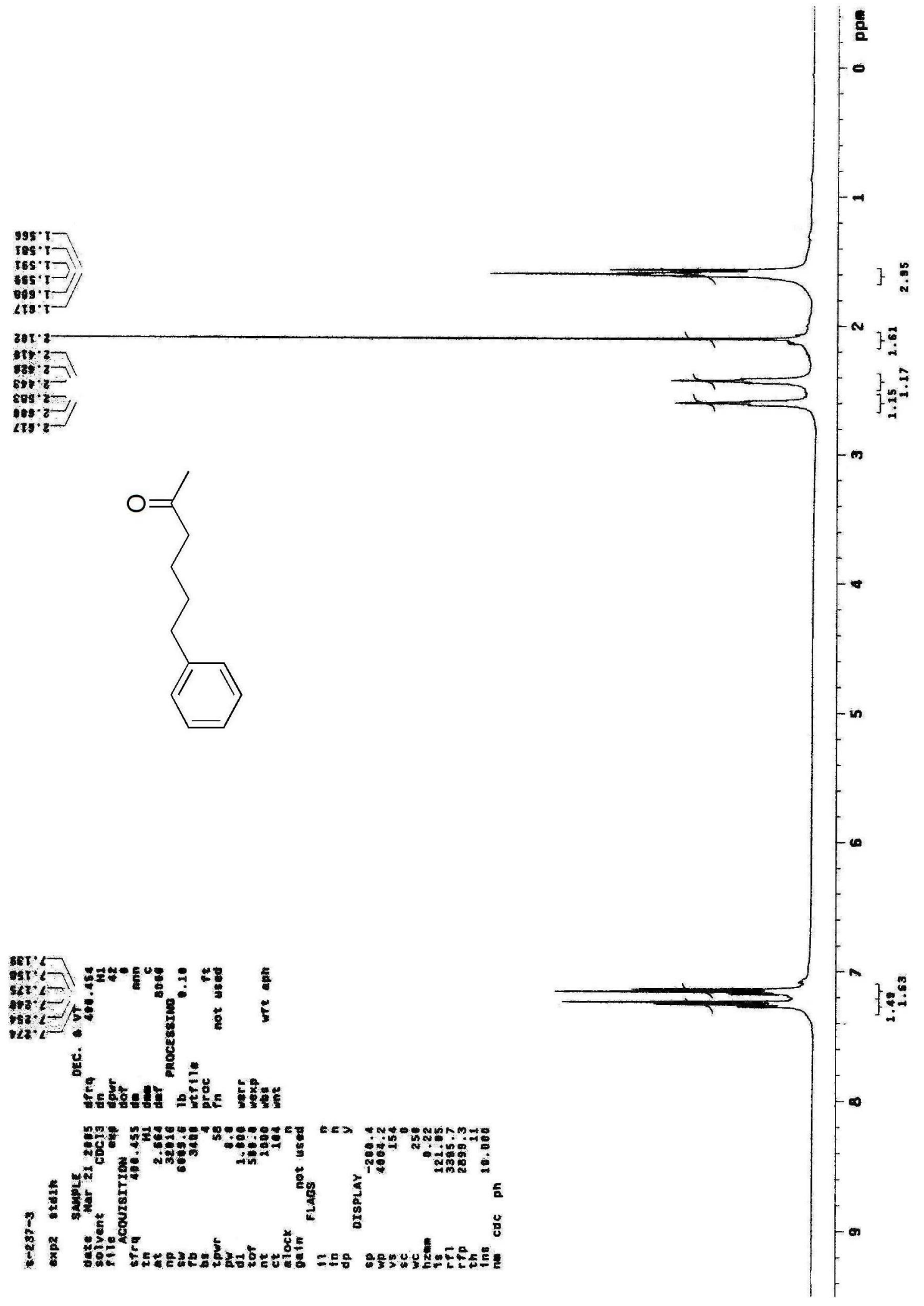




\section{${ }^{13} \mathrm{C}$ NMR spectrum of $3 \mathrm{k}$}
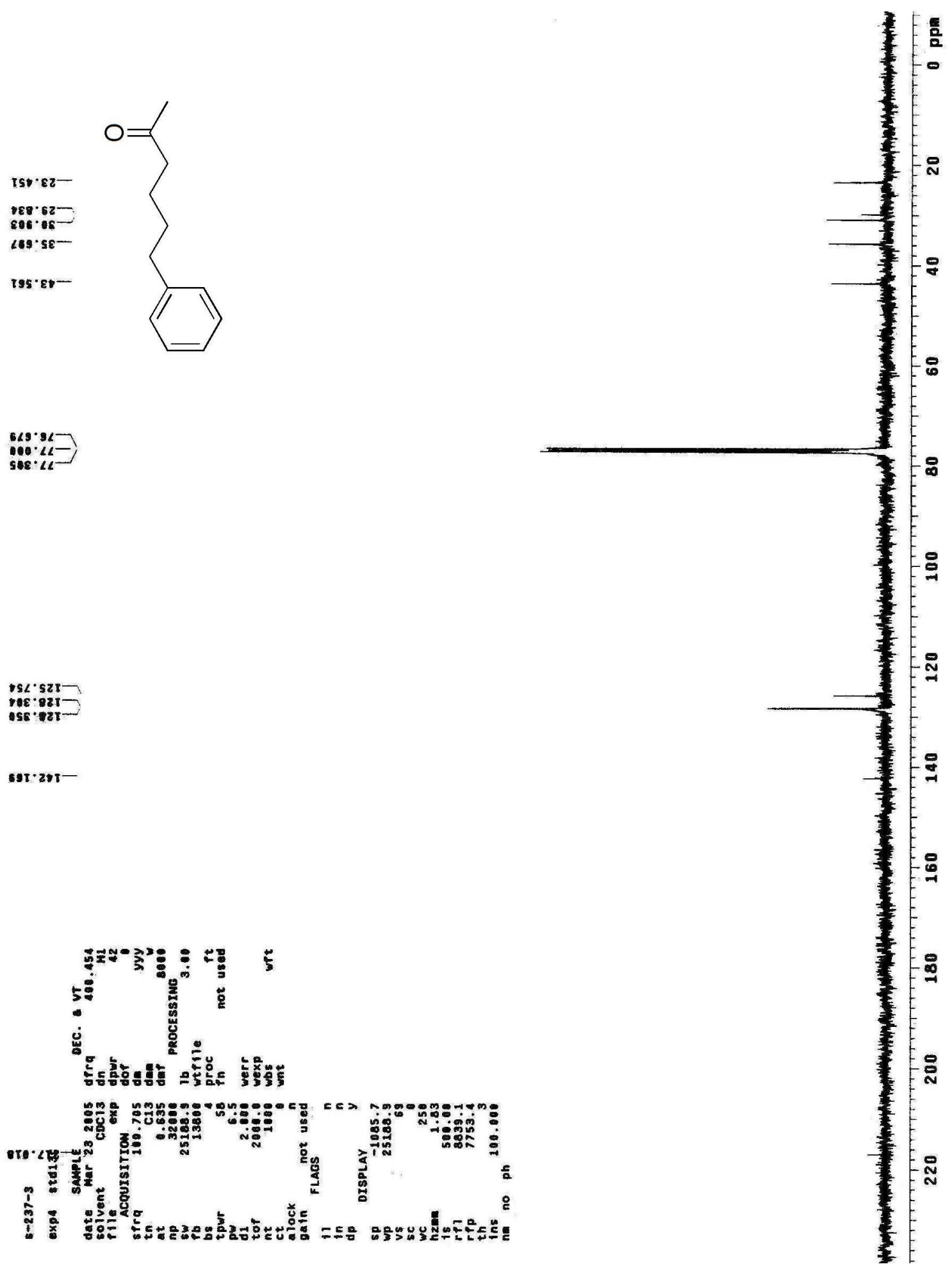
${ }^{\text {I }}$ H NMR spectrum of 31

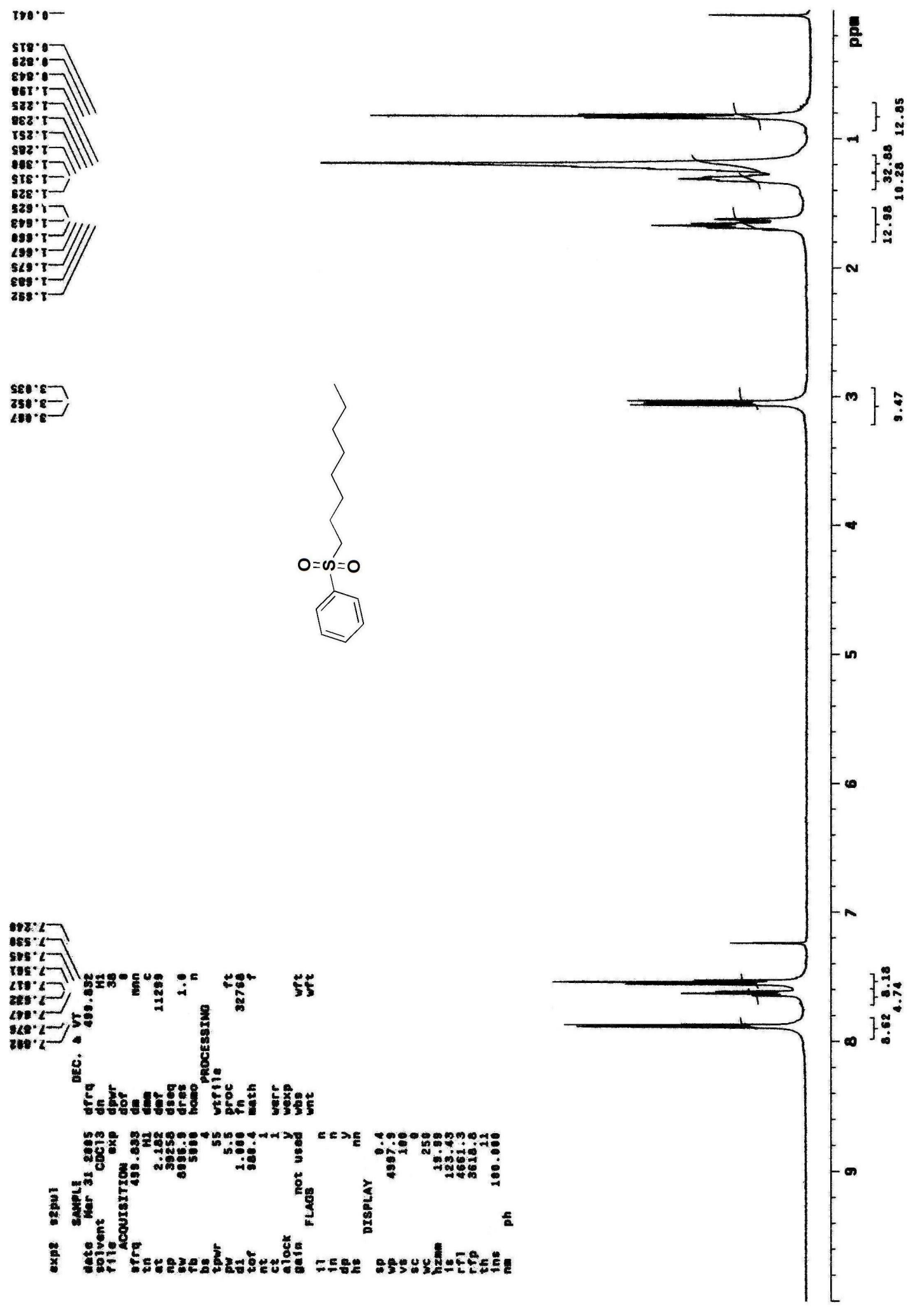




\section{${ }^{13}$ C NMR and DEPT spectrum of 31}

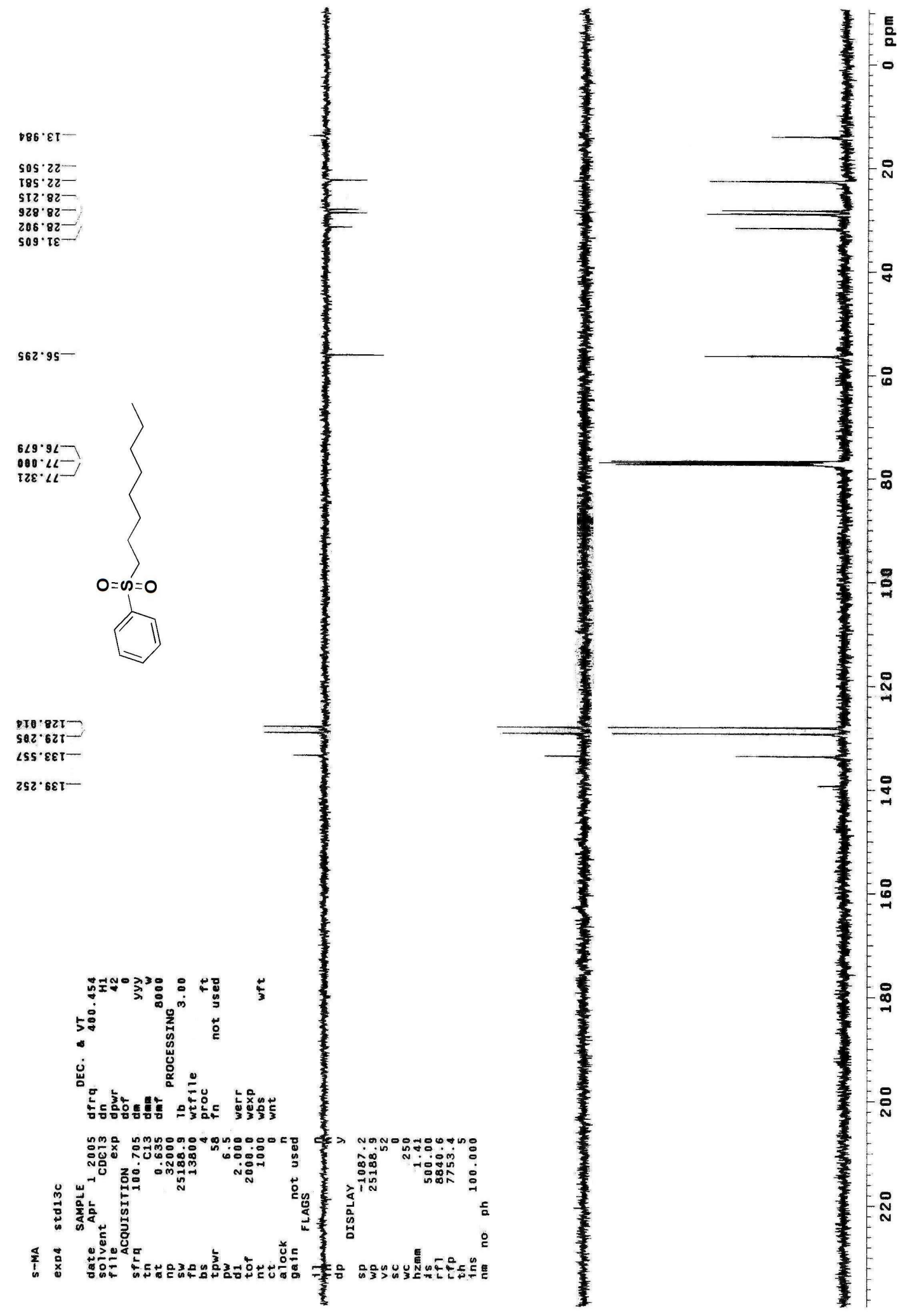




\section{${ }^{\text {I}}$ H NMR spectrum of $\mathbf{3 m}$}
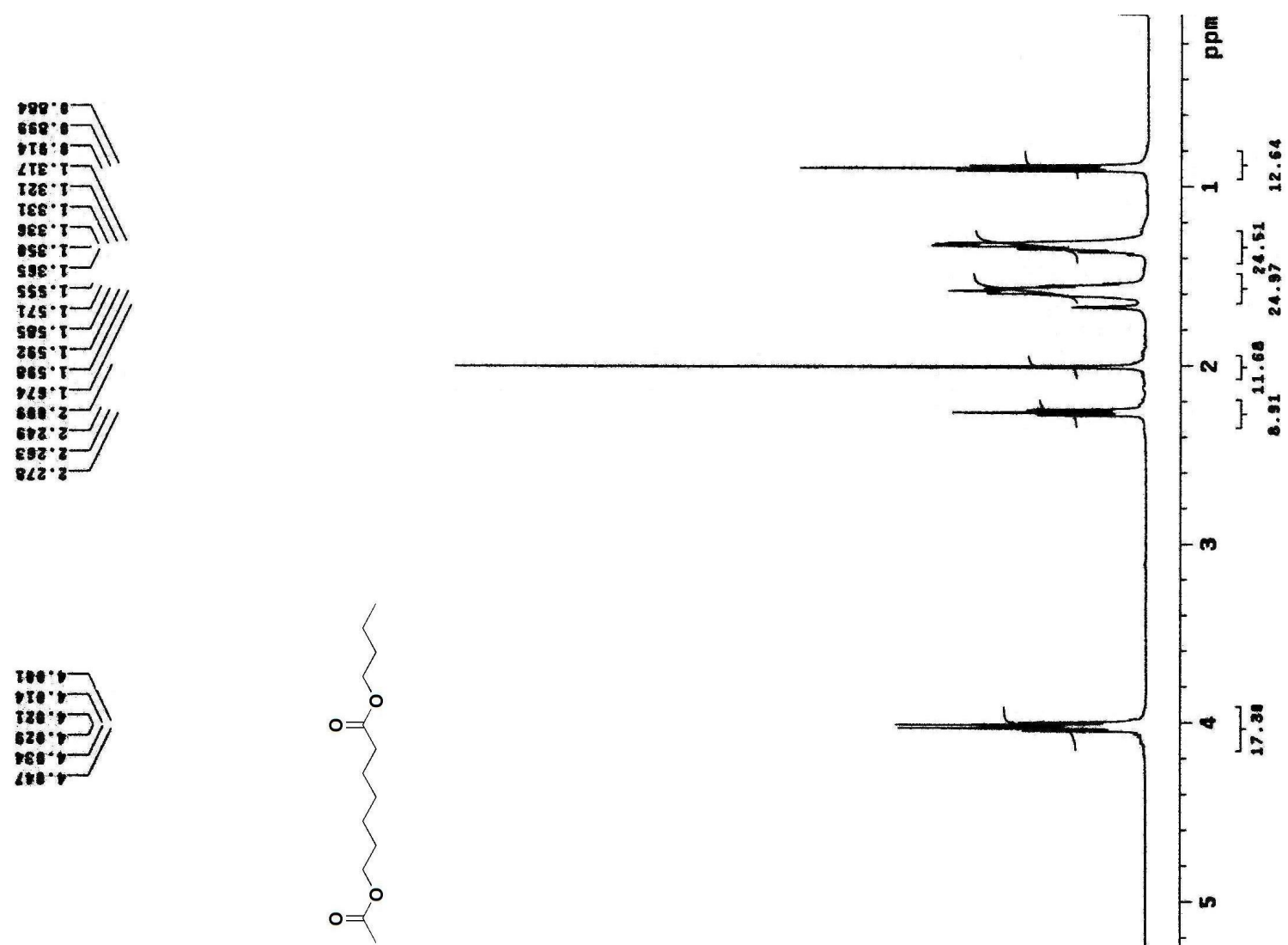

$m \cdot 2-$
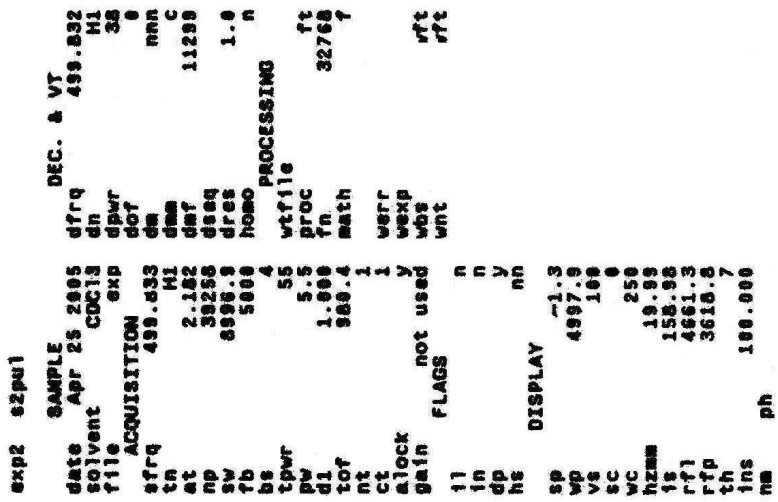


\section{${ }^{13} \mathrm{C}$ NMR and DEPT spectrum of $3 \mathrm{~m}$}
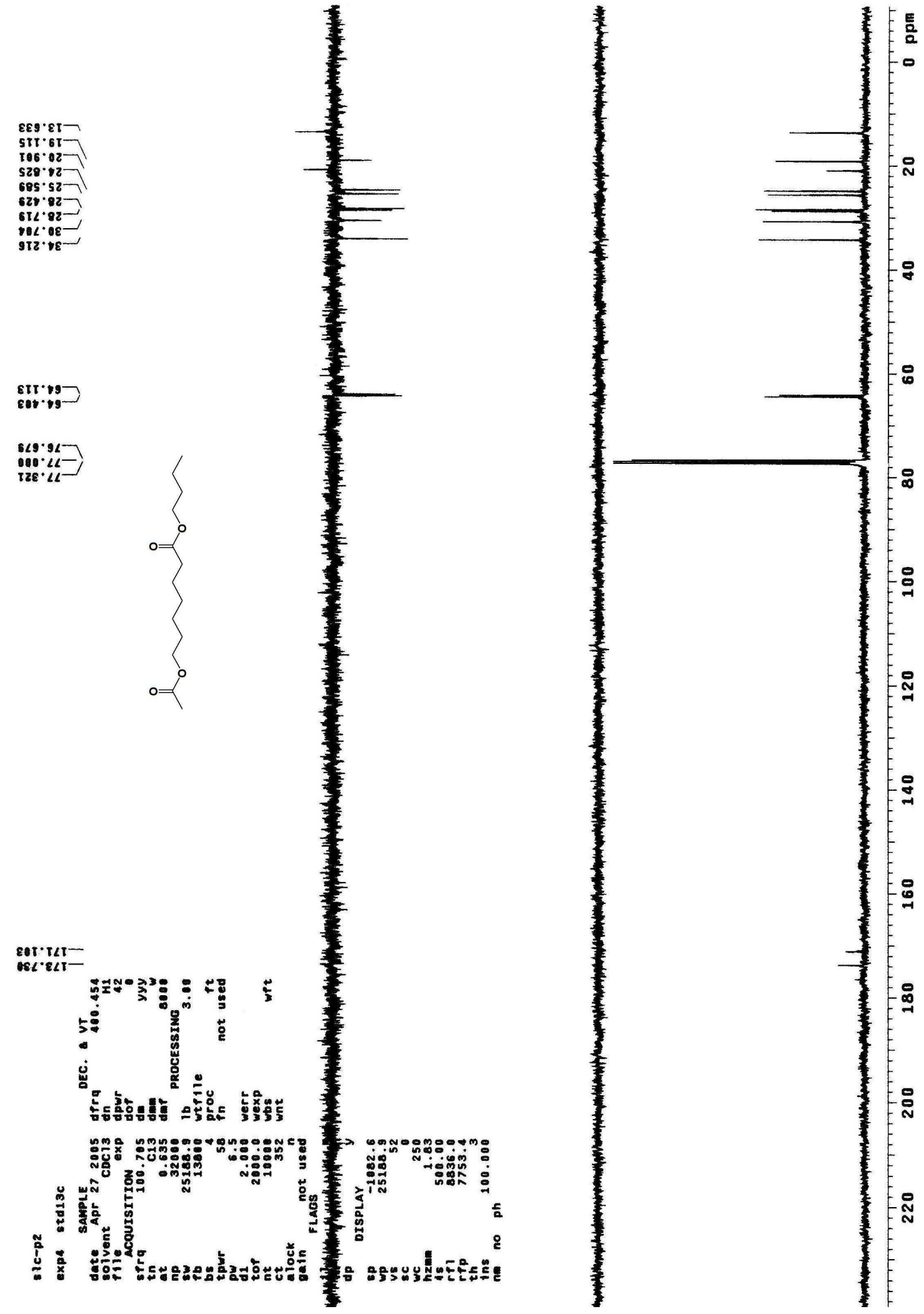